\title{
OH spectral evolution of oxygen-rich late-type stars ${ }^{\star}$
}

\author{
S. Etoka ${ }^{1}$ and A. M. Le Squeren ${ }^{2}$ \\ 1 Jodrell Bank Observatory, University of Manchester, Macclesfield, Cheshire SK11 9DL, UK \\ 2 GEPI, Observatoire de Paris-Meudon, 5 place J. Janssen, 92195 Meudon Cedex, France
}

Received 10 September 2003 / Accepted 27 January 2004

\begin{abstract}
We investigated the main-line spectral evolution with shell thickness of oxygen rich AGB stars. The study is based on a sample of 30 sources distributed along the IRAS colour-colour diagram. The sources were chosen to trace the Miras with thick shells and the whole range of $\mathrm{OH} / \mathrm{IR}$ stars. The Miras exhibit a $1665 \mathrm{MHz}$ emission strength comparable to that at $1667 \mathrm{MHz}$. Even though the Miras of the study have quite thick shells, their spectral characteristics in both main lines attest to a strong heterogeneity in their $\mathrm{OH}$ shell with, in particular, the presence of significant turbulence and acceleration. The expansion velocity has been found to be about the same at 1665 and $1667 \mathrm{MHz}$, taking into account a possible velocity turbulence of 1-2 $\mathrm{km} \mathrm{s}^{-1}$ at the location of the main-line maser emission. An increase in the intensity ratio 1667/1665 with shell thickness has been found. A plausible explanation for such a phenomenon is that competitive gain in favour of the $1667 \mathrm{MHz}$ line increases when the shell is getting thicker. There is an evolution in the spectral profile shape with the appearance of a substantial inter-peak signal when the shell is getting thicker. Also, inter-peak components are found and can be as strong as the external standard peaks when the shell is very thick. This trend for an increase of the signal in between the two main peaks is thought to be the result of an increase of the saturation with shell thickness. All sources but two - a Mira and an OH/IR star from the lower part of the colour-colour diagram - are weakly polarized. The strong polarization observed for those two particular objects is thought to be the result of perturbations in their shells.
\end{abstract}

Key words. stars: AGB and post-AGB - stars: circumstellar matter - masers - line: profiles - stars: mass-loss stars: evolution

\section{Introduction}

The standard model proposed by Reid et al. (1977) explains convincingly the double-peaked profile usually observed for Miras and OH/IR objects, particularly at $1612 \mathrm{MHz}$. It also explains the general features of the circumstellar envelopes. Nevertheless, many stars do not have such a simple $\mathrm{OH}$ maser profile. In particular, in the main lines, the peaks are often broad and consist of many components. To explain these more complex profiles new models have been suggested.

Alcock \& Ross (1986) have shown that it is possible to change the profile shape for a partially or fully saturated maser when the masering region becomes sufficiently thick. When that happens, the profiles consist of broader peaks and a signal is seen at the velocity of the star. This is in agreement with the spectral profiles observed for Miras and OH/IR objects, but the inferred internal and external radii disagree with the MERLIN and VLA interferometric observations. To overcome this discrepancy, Alcock \& Ross suggest that the mass loss is not a continuous and uniform process but rather consists of ejection of blobs of material in random directions such that the symmetrical geometry is preserved. The model calculation results

\footnotetext{
Send offprint requests to: S. Etoka,

e-mail: setoka@jb.man.ac.uk

* Appendices A-C are only available in electronic form at http://www. edpsciences.org
}

of Collison \& Nedoluha (1995) agree with the conclusions by Alcock \& Ross that the observed $\mathrm{OH}$ profile cannot be produced by the standard model description of a smooth, spherically symmetric, steady windlike mass loss. The assumption of a non-smooth shell is justified by the interferometric maps themself. Indeed, with the increase in interferometer resolution the more detailed maps revealed that the $\mathrm{OH}$ maser emission is located in clumps (Welty et al. 1987; Chapman et al. 1991) and/or the shell shows evidence for deviation from spherical symmetry (Diamond et al. 1985; Bowers et al. 1989).

Evidence for acceleration in the $\mathrm{OH}$ shell has been found for many stars (Chapman et al. 1994; Etoka \& Le Squeren 1996, 1997; Szymczak et al. 1998; Richards et al. 1999). The importance of acceleration in the shape of the spectral profile is underlined by the work of Chapman \& Cohen (1985). According to the value of the logarithmic velocity gradient $\epsilon$, the shape of the observed line profile largely varies as follows: (1) for $\epsilon \rightarrow 0$ the classical double-peaked profile is observed; (2) for $0<\epsilon<1$ an inter-peak signal can be seen; (3) for $\epsilon \simeq 1$ a plateau-shaped profile is obtained and finally (4) for $\epsilon>1$ the profile is reduced to a single peak centred on the stellar velocity. Thus, many spectral profiles resembling those observed can be modelled by introducing acceleration in the $\mathrm{OH}$ shell.

Based on the IRAS measurements at 12,25 and $60 \mu \mathrm{m}$, Olnon et al. (1984) discovered the existence of a continuous sequence from Miras to $\mathrm{OH} / \mathrm{IR}$ objects in the so-called 
colour-colour diagram which plots the colour indices [60-25] vs. [25-12]. This sequence has been analysed in terms of shell thickness by van der Veen \& Habing (1988) and van der Veen \& Rugers (1989). These studies lead to a commonly accepted interpretation that it is an evolutionary track followed by intermediate-mass stars. In this scenario, Miras are the progenitors of $\mathrm{OH} / \mathrm{IR}$ stars involving among other characteristics an increase of their mass-loss rate and expansion velocity with time. Nonetheless, the initial mass of the star could play a role in the setting up of that sequence. Based on an alternative model for the evolution of AGB stars proposed by Epchtein et al. (1990), Lépine et al. (1995) proposed that the IRAS colour-colour sequence could be a sequence of mass.

The study presented here deals with the spectral profiles in both main lines of Miras and OH/IR stars distributed along the colour-colour diagram. The main aim is to determine whether there is a relation between the spectral profile and the thickness of the circumstellar shell. The next section presents the observations and selection criteria. Section 3 and Appendices A-C present the results concerning the individual stars and a comparison with previous observations. The discussion is presented in Sect. 4 and the final conclusions in Sect. 5.

\section{Observations}

The $\mathrm{OH}$ observations were carried out between March and December 1994 with the Nançay transit radiotelescope. The half-power beamwidth of the radiotelescope at $1.6 \mathrm{GHz}$ is $3.5^{\prime}$ in $\alpha$ by $19^{\prime}$ in $\delta$. The system temperature was about $50 \mathrm{~K}$. A frequency switching mode was used. The autocorrelator was divided into 4 banks of 256 channels each, allowing simultaneous observations of the two $\mathrm{OH}$ main lines (i.e., 1665 and $1667 \mathrm{MHz}$ ) in both left- and right-handed circular polarizations (LHC, RHC). A bandwidth of $0.78 \mathrm{MHz}$ was used, leading to a velocity resolution of $0.56 \mathrm{~km} \mathrm{~s}^{-1}$. The ratio of flux to antenna temperature was $1.1 \mathrm{Jy} \mathrm{K}^{-1}$ at $0^{\circ}$ declination. Each observation lasted about one hour providing a mean rms of $0.035 \mathrm{~K}$. All the radial velocities given hereafter are relative to the Local Standard of Rest (LSR).

\subsection{Selection criteria}

All the sources presented here had already been positively detected at $1667 \mathrm{MHz}$ and a few of them at $1665 \mathrm{MHz}$ too. We looked for $1665 \mathrm{MHz}$ emission for a possible signature of circumstellar shell evolution. Figure 1 shows the colour-colour diagram of the selected sources. The colour indices [25-12] and [60-25] used here were calculated according to the following relation:

$\left[\lambda_{1}-\lambda_{2}\right]=\log \left(\frac{v_{1} S_{v}\left(\lambda_{1}\right)}{v_{2} S_{v}\left(\lambda_{2}\right)}\right)$

similar to that used by Sivagnanam et al. (1990).

The selected objects were intended to cover the far righthand side of the region labelled "Mira-like" by Sivagnanam et al. (1990) and the areas labelled LSDD and $R_{2}$ by

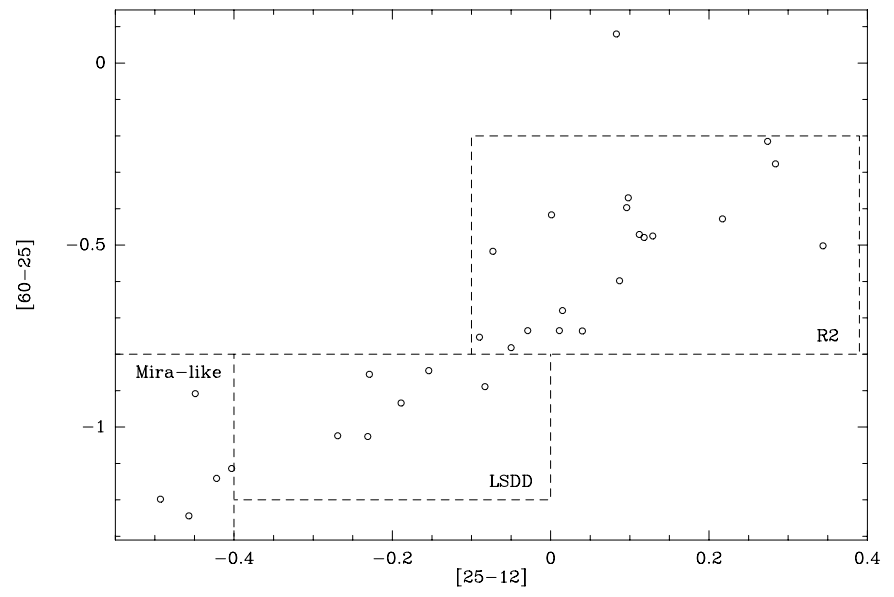

Fig. 1. [60-25] vs. [25-12] colour-colour diagram of the sources of the study.

David et al. (1993a) for the OH/IR sources. These areas are respectively delimited as follows:

$-0.50<[25-12]<-0.40$

$-1.30<[60-25]<-0.80$

$-0.40<[25-12]<+0.00$

$-1.20<[60-25]<-0.80$

and

$-0.10<[25-12]<+0.40$

$-0.80<[60-25]<-0.20$.

These areas are covered by the regions labelled IIIa (i.e., variable stars with more evolved $O$-rich circumstellar shells), IIIb (i.e., variable stars with thick $O$-rich circumstellar shells) and IV (i.e., variable stars with very thick $O$-rich circumstellar shells) by van der Veen \& Habing (1988) and van der Veen \& Rugers (1989).

The observations by David et al. (1993a) were done at 1612 and $1667 \mathrm{MHz}$, and those of Sivagnanam et al. (1990) at $1667 \mathrm{MHz}$ only. Both sets of observations were carried out with the Nançay radiotelescope with the same resolution as the present work, allowing a direct comparison of the profile shapes. Observations made by David et al. (1993a) for the stars of interest were taken over a period of 5.5 years from mid-1986 to the early 1992. Those of Sivagnanam et al. (1990) were all obtained in July 1986 except that of IRAS $20547+0247$ which was obtained in May 1987.

\section{Results: General comments}

Table 1 presents the main characteristics of the sources of the study. Columns 1 to 10 give the name of the source, its galactic coordinates, its LRS class, its IRAS flux at 12, 25 and $60 \mu \mathrm{m}$, its [25-12] and [60-25] colour indices and finally the name under which it is also known. The table was divided such that the three parts correspond to the three regions "Mira-like", LSDD and $R_{2}$ defined by the colour indices given in (2)-(4) respectively. 
Table 1. Galactic positions, IRAS flux at 12,25 and $60 \mu \mathrm{m}$ for the sources of the study.

\begin{tabular}{|c|c|c|c|c|c|c|c|c|c|}
\hline Iras name & $\begin{array}{r}l \\
\operatorname{deg}\end{array}$ & $\begin{array}{r}b \\
\operatorname{deg}\end{array}$ & $\begin{array}{l}\text { LRS } \\
\text { class }\end{array}$ & $\begin{array}{r}S_{12 \mu \mathrm{m}} \\
\mathrm{Jy}\end{array}$ & $\begin{array}{r}S_{25 \mu \mathrm{m}} \\
\mathrm{Jy}\end{array}$ & $\begin{array}{r}S_{60 \mu \mathrm{m}} \\
\mathrm{Jy}\end{array}$ & {$[25-12]$} & [60-25] & Other name \\
\hline $15226-3603$ & 334.7 & 17.0 & 28 & 166.40 & 121.10 & 16.58 & -0.457 & -1.244 & \\
\hline $15262+0400$ & 8.1 & 45.8 & 27 & 46.15 & 30.87 & 4.70 & -0.493 & -1.198 & \\
\hline 20396-0826 & 38.3 & -28.4 & 69 & 11.71 & 9.23 & 1.60 & -0.422 & -1.141 & XX Aqr \\
\hline $20547+0247$ & 51.4 & -26.1 & 32 & 45.52 & 33.76 & 10.02 & -0.449 & -0.908 & U Equ \\
\hline $22525+6033$ & 109.2 & 1.1 & 24 & 112.10 & 92.30 & 17.05 & -0.403 & -1.114 & \\
\hline $17482-2824$ & 1.1 & -0.8 & 42 & 428.70 & 480.40 & 109.10 & -0.269 & -1.024 & OH $1.1-0.8$ \\
\hline $17505-3143$ & 358.5 & -2.9 & 34 & 47.60 & 69.51 & 23.82 & -0.154 & -0.845 & \\
\hline $19075+0921$ & 43.4 & 0.2 & 12 & 133.00 & 163.70 & 54.91 & -0.229 & -0.855 & \\
\hline $19161+2343$ & 57.1 & 5.1 & 31 & 112.20 & 137.20 & 31.04 & -0.231 & -1.026 & \\
\hline $19190+1128$ & 46.6 & -1.3 & 32 & 27.13 & 36.60 & 10.22 & -0.189 & -0.934 & \\
\hline $19244+1115$ & 47.1 & -2.5 & 28 & 1346.00 & 2314.00 & 717.70 & -0.083 & -0.889 & IRC 10420 \\
\hline $17220-2448$ & 1.0 & 6.1 & & 5.35 & 9.06 & 3.84 & -0.090 & -0.753 & \\
\hline $17317-3331$ & 354.9 & -0.5 & 79 & 104.00 & 291.50 & 234.50 & 0.129 & -0.475 & \\
\hline 17385-3332 & 355.6 & -1.7 & & 2.88 & 13.25 & 10.01 & 0.344 & -0.502 & \\
\hline 17392-3319 & 355.9 & -1.7 & 37 & 19.12 & 37.29 & 16.49 & -0.029 & -0.735 & \\
\hline $17411-3154$ & 357.3 & -1.3 & & 1262.00 & 2723.00 & 1365.00 & 0.015 & -0.680 & \\
\hline $17418-2713$ & 1.4 & 1.0 & 79 & 14.95 & 51.28 & 45.94 & 0.217 & -0.428 & $\mathrm{OH} 1.3+1.0$ \\
\hline $17550-2120$ & 8.0 & 1.4 & & 5.38 & 21.08 & 30.81 & 0.274 & -0.215 & $\mathrm{OH} 8.0+1.4$ \\
\hline $18033-2111$ & 9.1 & -0.2 & & 9.75 & 20.84 & 9.20 & 0.011 & -0.735 & \\
\hline $18135-1456$ & 15.7 & 0.8 & 79 & 31.02 & 124.40 & 157.60 & 0.284 & -0.277 & OH $15.7+0.8$ \\
\hline 18198-1249 & 18.3 & 0.4 & & 15.56 & 42.54 & 33.91 & 0.118 & -0.479 & $\mathrm{OH} 18.3+0.4$ \\
\hline $18257-1000$ & 21.5 & 0.5 & 39 & 46.32 & 120.50 & 115.90 & 0.096 & -0.397 & OH $21.3+0.4$ \\
\hline $18348-0526$ & 26.5 & 0.6 & & 359.80 & 633.80 & 463.00 & -0.073 & -0.517 & OH $26.5+0.6$ \\
\hline 18432-0149 & 30.7 & 0.4 & & 25.06 & 52.32 & 48.09 & 0.001 & -0.417 & OH $30.7+0.4$ \\
\hline 18460-0254 & 30.1 & -0.7 & & 111.10 & 279.90 & 806.90 & 0.083 & 0.080 & OH $30.2-0.7$ \\
\hline 18488-0107 & 31.9 & -0.4 & & 16.45 & 42.98 & 44.03 & 0.098 & -0.370 & OH $32.0-0.5$ \\
\hline $19065+0832$ & 42.6 & 0.1 & 39 & 20.22 & 51.52 & 31.18 & 0.087 & -0.598 & $\mathrm{OH} 42.6+0.0$ \\
\hline $19254+1631$ & 51.8 & -0.2 & 39 & 16.57 & 44.67 & 36.27 & 0.112 & -0.471 & OH $51.8-0.2$ \\
\hline $19352+2030$ & 56.4 & -0.3 & 03 & 42.89 & 97.88 & 43.12 & 0.040 & -0.736 & OH 56.4-0.3 \\
\hline $22177+5936$ & 104.9 & 2.4 & 38 & 123.20 & 228.90 & 90.65 & -0.050 & -0.782 & OH $104.9+2.4$ \\
\hline
\end{tabular}

The results concerning the spectral profile changes of the individual sources in the three regions of concern are presented in Appendix A.

Figure B.1 presents the spectral profiles of each source at 1665 and $1667 \mathrm{MHz}$ in both circular polarizations. As IRAS 18198-1249, IRAS 18257-1000, IRAS 18488-0107, IRAS 19065+0832, IRAS 22177+5936 and IRAS $19075+0921$ had already been detected before the work of David et al. (1993a), their spectra were not displayed by the previously cited authors. The spectral profiles of the 5 first cited sources are given in Dickinson \& Turner (1991) and that of IRAS $19075+0921$ is given in Le Squeren et al. (1992). Therefore, for these 6 sources, the spectral shape changes were determined from the comparison of our observations with those of the two papers mentioned, while for the other sources, comparison has been made with those of David et al. for the OH/IR stars and Sivagnanam et al. (1990) for the Miras. All the sources of the $R_{2}$ region were also observed at $1612 \mathrm{MHz}$ by David et al. (1993b) and quite a few by Te Lintel et al. (1991). Three, out of the six sources of the LSDD region were observed at $1612 \mathrm{MHz}$ by Te Lintel et al. (1991). Finally, all the sources of the "Mira-like" region were observed at 1667 and $1612 \mathrm{MHz}$ by Sivagnanam et al. (1990).

Tables C. 1 and C. 2 present the results of the spectral measurements done at 1665 and $1667 \mathrm{MHz}$ respectively. As for Table 1, these tables were divided into three parts corresponding to the three regions of concern. Columns 1 to 16 give the name of the source, a comment about its profile shape in the 
case of a non "standard" double-peaked profile, the velocity of the blue- and red-shifted peaks and the velocity of any interpeak component in both circular polarizations, the stellar and expansion velocities inferred, the flux intensity of the blue- and red-shifted peak and that of any inter-peak component in both circular polarizations (i.e., measured respectively at the velocity given in Cols. 3 to 8 ) respectively. For the profile shape we distinguish between 4 different categories: "standard" type spectrum but exhibiting inter-peak signal ("s"), plateau profile spectrum ("p"), spectrum with more than two well detached peaks (i.e., "standard" profile plus an internal feature, "ci" where "internal" means in between the two standard peaks) and finally spectrum showing only a single peak centred at or about the stellar velocity ("c"). For the two last categories, the characteristics of the signal are given in Cols. 7, 8, 15, and 16.

David et al. (1993a) used the 1985 IRAS fluxes while we used the corrected 1987 fluxes. Thus, IRAS 18460-0254 which was initially in the $R_{2}$ region of David et al. is now outside this box due to a notable difference in its [60-25] colour index when calculated with or without correction (cf. Fig. 1). Nevertheless, its [25-12] colour index is hardly affected by the application of the IRAS correction. Since this index is the most relevant in terms of mass loss and shell thickness dependence, we decided to incorporate this source in the statistics of the $R_{2}$ region.

\section{Discussion}

\subsection{The "Mira-like" sample}

The area corresponding to the $\mathrm{OH}$-emitter Miras contains five objects which, according to their location in the colour-colour diagram, have the thickest shells of their category. None of the five sources exhibits a standard spectral profile either at 1665 or $1667 \mathrm{MHz}$ and only one source shows a double-peaked profile characterized by two unevenly broad and spiky peaks. This source has the smallest [25-12] colour index value of the sample. All the sources show quite irregular spectral profile shapes. This tendency is also clearly observable in the spectral profiles obtained by Sivagnanam et al. (1990, their Fig. 2). Such a strong departure from the double-peaked spectral profile indicates a strong heterogeneity in the physical and dynamical conditions ruling over the whole $\mathrm{OH}$ shell even though those Miras have quite thick shells.

All the sources show $\mathrm{OH}$ variability. Among the five sources selected, four of them were primarily successfully detected in the satellite line at $1612 \mathrm{MHz}$ and we detected $1665 \mathrm{MHz}$ emission from all of them. For most of the sources of the "Mira-like" sample positively detected by Sivagnanam et al. (1990) as OH-emitters, when $1612 \mathrm{MHz}$ emission was detected its intensity was comparable to or greater than that at $1667 \mathrm{MHz}$. Such a trend in the strengthening of the $1612 \mathrm{MHz}$ emission over that of the main lines is expected when the shell is getting thick enough (cf. Lewis 1989; David et al. 1993b; Habing 1996). The sample selected here (i.e., $-0.50<[25-12]<-0.40$ ) is such that it fulfils the physical conditions for the $1612 \mathrm{MHz}$ and the main-line emissions to be of similar strength. IRAS 15226-3603 was one of the rare sources not detected positively at $1667 \mathrm{MHz}$, nor did we detect any $1665 \mathrm{MHz}$ emission from it. Since this source was detected positively by Sivagnanam et al. earlier, our nondetection is most likely due to the fact that the source was at its $\mathrm{OH}$ minimum at the time of our observations. Finally, one source (IRAS 22525+6033) appeared to be strongly polarized in both main lines. This source exhibits the strongest $1665 \mathrm{MHz}$ emission and the greatest expansion velocity of its group: about $10 \mathrm{~km} \mathrm{~s}^{-1}$. Thus, this source is located in the narrow zone where both Miras and OH/IR stars can be found in the "period-OH expansion velocity" diagram as given by Sivagnanam et al. (1989).

\subsection{The $O H / I R$ sample of the $L S D D$ region}

This sample of six sources presents the highest rate of doublepeaked spectral profiles at $1667 \mathrm{MHz}(>50 \%)$ with very faint or non-existent inter-peak emission. Four of the six sources (i.e., $67 \%$ ) were detected at $1665 \mathrm{MHz}$ at the time of the observations. Nevertheless, none of the sources shows a "classical" double-peaked profile at that frequency. In addition, the $1665 \mathrm{MHz}$ emission for the sources of this sample is very faint. Only IRAS $19075+0921$ shows a significant flux density at $1665 \mathrm{MHz}$ (i.e., comparable to that observed at $1667 \mathrm{MHz}$ ). This particular source also shows strong polarization in both main lines and has the smallest expansion velocity of the whole sample, with a value of $7.7 \mathrm{~km} \mathrm{~s}^{-1}$. Consequently, like IRAS $22525+6033$, IRAS $19075+0921$ is located in the zone where Miras and OH/IR stars coexist in the "period-OH expansion velocity" diagram of Sivagnanam et al. (1989).

\subsection{The $\mathrm{OH} / \mathrm{IR}$ sample of the $\mathrm{R}_{2}$ region}

The $R_{2}$ sample is composed of nineteen OH/IR stars. $84 \%$ of the stars of this group show at least two peaks in their $1667 \mathrm{MHz}$ spectral profile with only 31.5\% showing a standard double-peaked profile (i.e., with no inter-peak signal). The percentage of stars having at least two peaks at $1665 \mathrm{MHz}$ is only $48 \%$. Five sources (i.e., 26\%) exhibit a single-peaked spectral profile and five other sources did not show any $1665 \mathrm{MHz}$ emission at all. 26\% of the sources that were positively detected at $1665 \mathrm{MHz}$ show an internal group of components with an intensity comparable or superior to that of the red- or blue-shifted peaks.

Among the whole set of the double-peaked profiles of this group of thicker $\mathrm{OH} / \mathrm{IR}$ stars, nine sources (i.e., 48\%) show an inter-peak signal at $1667 \mathrm{MHz}$. Sun (1991) has modelled the evolution of the $1612 \mathrm{MHz}$ double-peaked profile when the mass-loss rate varies with time. The author shows that the interpeak signal at $1612 \mathrm{MHz}$ increases with the mass-loss rate. Our results show that this property also applies to the $1667 \mathrm{MHz}$ emission. Also, the existence of an inter-peak signal is related to the degree of saturation (Fix 1978). Therefore, the increased number of inter-peak signals observed in the profiles of these thicker OH/IR stars is quite probably a signature of greater maser saturation at $1667 \mathrm{MHz}$. 


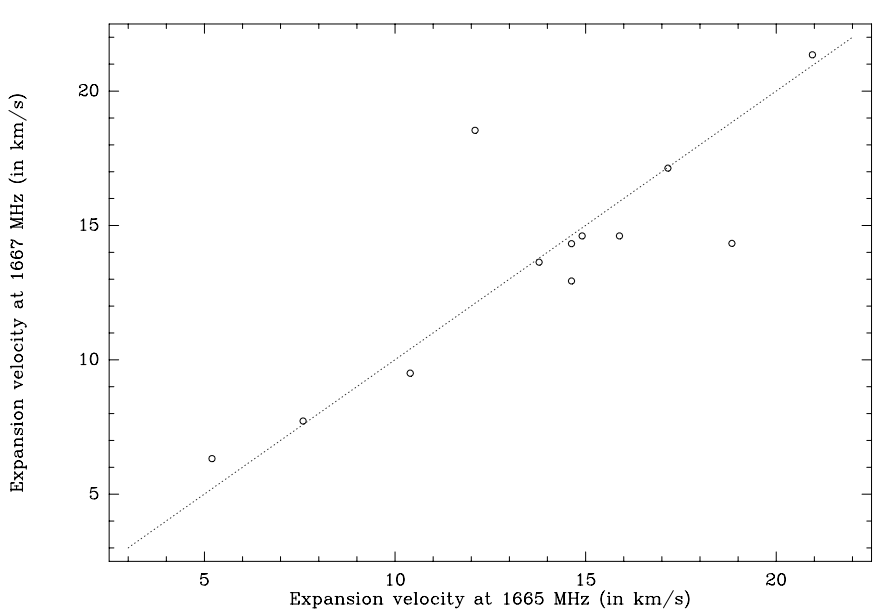

Fig. 2. Expansion velocity at $1667 \mathrm{MHz}$ versus that at $1665 \mathrm{MHz}$.

\subsection{Spectral profile shape as a function of the shell thickness}

Considering the three groups together, it stands out that the spectral profiles with inter-peak signal as strong as that of the red- or blue-shifted peaks are only observed for OH/IR sources at $1665 \mathrm{MHz}$. OH/IR stars with inter-peak signal at $1667 \mathrm{MHz}$ are found in both the LSDD and the $R_{2}$ regions. Except IRAS $19352+2030$ which shows very faint blue- and redshifted peaks and a very strong inter-peak component in both main lines, the sources show a faint $1667 \mathrm{MHz}$ inter-peak signal which can be slightly polarized. Spectral profiles showing a well-detached inter-peak component at $1665 \mathrm{MHz}$ arise from $\mathrm{OH} / \mathrm{IR}$ stars belonging to the upper part of the $R_{2}$ region, that is, from the stars which have a very thick shell. The "plateau" profiles are only observed at $1665 \mathrm{MHz}$, but in all three groups. It can be particularly pronounced for some objects (cf. IRAS 17411-3154). Such a profile shape generally attests to significant acceleration (i.e., Chapman \& Cohen 1985 for a velocity gradient $\epsilon \simeq 1$ ). This confirms that the $1665 \mathrm{MHz}$ emissive zone indeed originates deeper inside the $\mathrm{OH}$ shell than the $1667 \mathrm{MHz}$ zone.

Figure 2 shows the correlation between the expansion velocity inferred at $1665 \mathrm{MHz}$ and that at $1667 \mathrm{MHz}$. This diagram uses the measurements from all the sources that showed at least two peaks in both main lines except IRAS 19244+1115 for which the expansion velocities inferred have to be taken with caution (cf. Appendix A.2). The total number of such sources is 12 , that is about $41 \%$ of the whole sample of 29 objects for this statistic. This corresponds respectively to 40,40 and $42 \%$ of the "Mira-like", LSDD and $R_{2}$ samples. The dotted line in the figure is the locus for $V_{1665 \mathrm{MHz}}=V_{1667 \mathrm{MHz}}$. Most of the sources have a similar expansion velocity in both main lines. At the location of the $\mathrm{OH}$ shell, some $10^{16} \mathrm{~cm}$ away from the central star, turbulence of $1-2 \mathrm{~km} \mathrm{~s}^{-1}$ is expected (Deguchi 1982). This could result in the broadening of the line wings and therefore explain most of the cases where $1665 \mathrm{MHz}$ profiles overshoot those of the $1667 \mathrm{MHz}$ line by such a value. One source, IRAS 17392-3319 from the $R_{2}$ region, shows an expansion velocity at $1665 \mathrm{MHz}$ greater by $4 \mathrm{~km} \mathrm{~s}^{-1}$ than that inferred at $1667 \mathrm{MHz}$. This may be due to the faintness of the

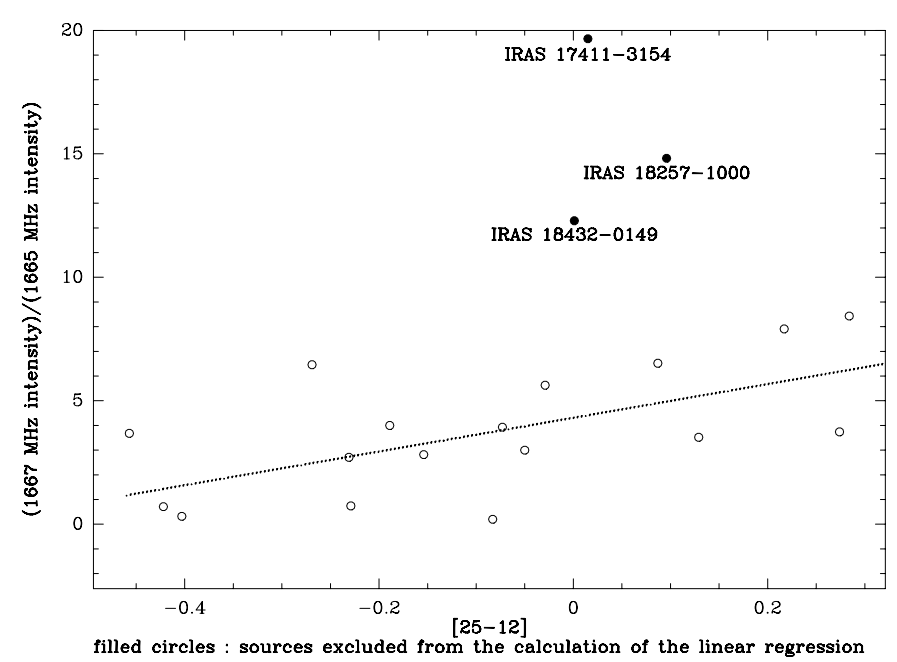

Fig. 3. (1667 MHz)/(1665 MHz) intensity ratio versus the colour index [25-12].

signal at $1665 \mathrm{MHz}$ leading to a bigger uncertainty in the exact location of the peak maxima.

Only four sources show an intensity at $1665 \mathrm{MHz}$ greater than that at $1667 \mathrm{MHz}$. These sources belong to the "Mira-like" and LSDD regions. None of the sources of the $R_{2}$ region show this peculiarity. We have investigated whether there was any correlation between the intensity observed in the main lines and the thickness of the shell. Taking all the sources showing both 1665 and $1667 \mathrm{MHz}$ emission, we have found that there is a relation between the ratio of the intensity observed in the two main lines and the [25-12] colour index. This relation is shown Fig. 3, where the dotted line shows the linear regression over all the sources except the three for which Intensity $_{1667} \gg$ Intensity $_{1665}$ (plotted with filled symbols). The linear regression is given by:

$\operatorname{Int}(1667 / 1665)_{i}=6.54 \times[25-12]_{i}+4.40$

where $\operatorname{Int}(1667 / 1665)_{i}$ is the ratio of the maximum intensity measured at 1667 and $1665 \mathrm{MHz}$ for the source " $i$ " and $[25-12]_{i}$ is its colour index.

Three sources do not lie on the relation given by Eq. (5). We do not think that this could be due to variability. Indeed, for one of the sources (IRAS 18432-0149) the $1665 \mathrm{MHz}$ emission faintness $(\sim 2 \sigma)$ could be responsible for the high $\operatorname{Int}(1667 / 1665)$ found but such an explanation is not plausible for the two other sources which showed reasonably strong $1665 \mathrm{MHz}$ emission at the time of the observations. On the other hand, it is noteworthy that the three sources not following the general trend have similar [25-12] indices: [0; 0.1].

Equation (5) shows that when the shell becomes thicker, the $1667 \mathrm{MHz}$ emission is favoured in comparison with that at $1665 \mathrm{MHz}$. Since the expansion velocity is about the same at 1665 and $1667 \mathrm{MHz}$ it is expected that the maser emission from both main lines should be found at similar distances from the star and possibly share the same regions of the $\mathrm{OH}$ shell. This hypothesis is supported by the maps of late-type stars obtained in VLBI (cf. Chapman et al. 1991, 1994; Szymczak et al. 1999). Therefore, a plausible explanation for such a trend is an 
increase of competitive gain in favour of the $1667 \mathrm{MHz}$ line when the shell becomes thicker.

This increase of the ratio of the $1667 \mathrm{MHz}$ emission over that at $1665 \mathrm{MHz}$ is in agreement with the fact that $1667 \mathrm{MHz}$ emission becomes relatively stronger and eventually dominant in the post-AGB stage (Zijlstra et al. 1989, 2001). The dominance of the $1667 \mathrm{MHz}$ emission at this stage of the stellar evolution is supported by theoretical studies (Field 1985; Field \& Gray 1988).

\subsection{The Mira-OH/IR sequence}

The Miras-OH/IR sequence seen in the IRAS colour-colour diagram results from the combination of various relations existing amongst the physical parameters of the central star and the circumstellar envelopes: (1) between the mass-loss rate and the period (Vassiliadis \& Wood 1993), (2) between the period and the expansion velocity (Dickinson \& Chaison 1973; Le Squeren et al. 1979), (3) between the OH luminosity and the flux at $25 \mu \mathrm{m}$ (Sivagnanam et al. 1989), (4) between the $\mathrm{OH}$ radius and the $\mathrm{OH}$ luminosity (Baud \& Habing 1983), (5) between the size of the maser regions and the mass-loss rate (predicted for the $\mathrm{H}_{2} \mathrm{O}$ masers by Cooke \& Elitzur 1985 and observed by Cohen 1987; Lane et al. 1987; and Yates \& Cohen 1994) and (6) between the mass-loss rate and the optical depth at $10 \mu \mathrm{m}$ (Bedijn 1988). This can by summarized as follows: the increase of the pulsation period, the expansion velocity, the radius of the circumstellar shell and the luminosity are all function of the mass-loss rate and the opacity of the shell.

The sequence observed in the colour-colour diagram may be either evolutionary or related to the stellar mass. If it is an evolutionary sequence the star should experience an increase of its mass-loss rate with time. If it is a mass sequence the mass loss should be connected somehow to the initial mass. Volk \& Kwok (1988) studied the IR spectral evolution of AGB stars. Their study shows that AGB stars that have a silicate signature at $10 \mu \mathrm{m}$ in absorption are mainly stars with an initial mass on the main sequence greater than $3 M_{\odot}$. This implies that the shell thickness depends on the initial mass, since only the stars massive enough will have a thick shell i.e. will become OH/IR stars. Also, through a study of the galactic distribution of $\mathrm{OH} / \mathrm{IR}$ stars according to their expansion velocity, Likkel (1989) found that the reddest OH/IR objects tend to have the highest initial masses. This latter observational result is in agreement with earlier work by Baud et al. (1981) who showed the existence of a relation between the expansion velocity and the initial mass of the central star.

We found that IRAS 22525+6033 and IRAS 19075-0921 belonging to the "Mira-like" group and to the LSDD group respectively show very similar characteristics: $1665 \mathrm{MHz}$ flux comparable to that at $1667 \mathrm{MHz}$, strong polarization over the whole velocity range of the spectrum in both main lines, similar $\mathrm{OH}$ expansion velocity values and [25-12] indices. This puts these two objects in the location where Miras and $\mathrm{OH} / \mathrm{IR}$ stars coexist both in the "period-OH expansion velocity" diagram and the colour-colour diagram, but they have a completely unusual set of spectral characteristics in comparison with the rest of the stars with similar expansion velocities and IRAS [25-12] fluxes. In particular, the very strong polarization observed over the whole spectral profile is clearly a sign of perturbation undergone throughout the whole $\mathrm{OH}$ circumstellar shell, which we interpret to be a signature that these objects are in the transition phase Mira $\rightarrow \mathrm{OH} / \mathrm{IR}$ star. Indeed, similar polarization characteristics in the $\mathrm{OH}$ shell have been observed (Bains et al. 2003; Etoka \& Diamond 2004) attesting to the importance of the role played by the magnetic field (in particular in the envelope shaping) in the transitional stages of evolved stars. Bearing in mind the existing relations between the initial mass and some of the actual physical properties of AGB stars such as their shell thickness or their expansion velocity, it is very likely that the IRAS colour-colour sequence is related to both the initial mass of the object and its evolutionary stage. A likely hypothesis is that the object arrives more or less high on the sequence and evolves through a part of it before expelling its shell entirely via an increasing mass loss.

\section{Conclusion}

Acceleration and turbulence can strongly alter the spectral profile shapes of circumstellar $\mathrm{OH}$ masers. This is well illustrated by the variety of spectral profiles observed for the "Mira-like" sample. When the shell gets thicker and less turbulent, the standard double-peaked profile settles. At that stage, saturation is the main cause of spectral profile alteration at $1667 \mathrm{MHz}$, causing an inter-peak signal to appear while plateau-shape or strong inter-peak components observed in the spectral profiles at $1665 \mathrm{MHz}$ still attest to the presence of acceleration. Even though the spectral profile characteristics show that the $1665 \mathrm{MHz}$ emission can be found deeper in the $\mathrm{OH}$ shell than the $1667 \mathrm{MHz}$ emission, the expansion velocity is found to be about the same in both main lines, taking into account a possible velocity turbulence of $1-2 \mathrm{~km} \mathrm{~s}^{-1}$ at the location of the main-line maser emission. It is then expected that the maser emission from both main lines should be found at similar distances from the star and possibly share the same regions of the $\mathrm{OH}$ shell. Also, the intensity ratio $1667 / 1665$ has been found to increase with the shell thickness. This can be explained as an increase of competitive gain in favour of the $1667 \mathrm{MHz}$ when the shell is becoming thicker. Finally, a Mira and an OH/IR star from the lower part of the colour-colour diagram show quite strong polarization while the rest of the sources are weakly polarized. Strong polarization can be the sign of dramatic changes in the shell itself. We suspect that the strong polarization observed in those two particular objects may be linked with their being in the phase of transition Mira $\rightarrow \mathrm{OH} / \mathrm{IR}$ star.

\section{References}

Alcock, C., \& Ross, R. R. 1986, ApJ, 305, 837

Bains, I., Gledhill, T. M., Yates, J. A., \& Richards, A. M. S. 2003, MNRAS, 338, 287

Baud, B., Habing, H. J., Matthews, H. E., \& Winnberg, A. 1981, A\&A, 95, 156

Baud, B., \& Habing, H. J. 1983, A\&A, 127, 73 
Bedijn, P. J. 1988, A\&A, 205, 105

Bowers, P. F. 1991, ApJS, 76, 1099

Bowers, P. F., Johnston, K. J., \& de Vegt, C. 1989, ApJ, 340, 479

Chapman, J. M., \& Cohen, R. J. 1985, MNRAS, 212, 375

Chapman, J. M., Cohen, R. J., \& Saikia, D. J. 1991, MNRAS, 249, 227

Chapman, J. M., Sivagnanam, P., Cohen, R. J., \& Le Squeren, A. M. 1994, MNRAS, 268, 475

Cohen, R. J. 1987, Circumstellar Matter, ed. I. Appenzeller, \& C. Jordan (Dordrecht: Reidel), Proc. IAU Symp., 122, 229

Collison, A. J., \& Nedoluha, G. E. 1995, ApJ, 442, 311

Cook, A. H. 1966, Nature, 211, 503

Cook, A. H. 1977, Celestial masers (Cambridge University Press)

Cooke, B., \& Elitzur, M. 1985, ApJ, 295, 175

David, P., Le Squeren, A. M., Sivagnanam, P., \& Braz, M. A. 1993a, A\&AS, 98, 245

David, P., Le Squeren, A. M., \& Sivagnanam, P. 1993b, A\&A, 277, 453

Deguchi, S. 1982, ApJ, 259, 634

Diamond, P. J., Norris, R. P., Rowland, P. R., Booth, R. S., \& Nyman, L.-A. 1985, MNRAS, 212, 1

Dickinson, D. F., \& Chaisson, E. J. 1973, ApJ, 181, L135

Dickinson, D. F., \& Turner, B. E. 1991, ApJS, 75, 1323

Epchtein, N., Le Bertre, T., \& Lepine, J. R. D. 1990, A\&A, 227, 82

Etoka, S., \& Le Squeren, A. M. 1996, A\&A, 315, 134

Etoka, S., \& Le Squeren, A. M. 1997, A\&A, 321, 877

Etoka, S., \& Diamond, P. 2004, MNRAS, 348, 34

Field, D. 1985, MNRAS, 217, 1

Field, D., \& Gray, M. D. 1988, MNRAS, 234, 353

Fix, J. D. 1978, ApJ, 223, L25

Goldreich, P., \& Scoville, N. 1976, ApJ, 205, 144

Habing, H. J. 1996, A\&ARv, 7, 97

Huggins, P. J., \& Glassgold, A. E. 1982, AJ, 87, 1828

Kholopov, P. N., Samus, N. N., Frolov, M. S., et al. 1985, General Catalog of Variable Stars, 4th ed., Moscow

Lanes, A. P., Johnston, K. J., Bowers, P. F., Spencer, J. H., \& Diamond, P. J. 1987, ApJ, 323, 756
Lépine, J. R. D., Ortiz, R., \& Epchtein, N. 1995, A\&A, 299, 453

Le Squeren, A. M., Baudry, A., Brillet, J., \& Darchy, B. 1979, A\&A, 72,39

Le Squeren, A. M., Sivagnanam, P., Dennefeld, M., \& David, P. 1992, A\&A, 254, 133

Lewis, B. M. 1989, ApJ, 338, 234

Likkel, L. 1989, ApJ, 344, 350

Te Lintel Hekkert, P., Caswell, J. L., Habing, H. J., Haynes, R. F., \& Norris, R. P. 1991, A\&AS, 90, 327

Olnon, F. M., Baud, B., Habing, H. J., et al. 1984, ApJ, 278, L41

Reid, M. J., Muhleman, D. O., Moran, J. M., Johnston, K. J., \& Schwartz, P. R. 1977, ApJ, 214, 60

Richards, A. M. S., Yates, J. A., \& Cohen, R. J. 1999, MNRAS, 306, 954

Sivagnanam, P., Braz, M. A., Le Squeren, A. M., \& Tran Minh, F. 1990, A\&A, 233, 112

Sivagnanam, P., Le Squeren, A. M., Foy, F., \& Tran Minh, F. 1989, A\&A, 211, 341

Sun, J. 1991, Chin. A\&A, 15/2, 197

Szymczak, M., Cohen, R. J., \& Richards, A. M. S. 1998, MNRAS, 297,1151

Szymczak, M., Cohen, R. J., \& Richards, A. M. S. 1999, MNRAS, 304, 877

Szymczak, M., Blaszkiewicz, L., Etoka, S., \& Le Squeren, A. M. 2001, A\&A, 379, 884

Ukita, N., \& Le Squeren, A. M. 1984, A\&A, 138, 343

Vassiliadis, E., \& Wood, P. R. 1993, ApJ, 413, 641

van der Veen, W. E. C. J., \& Habing, H. J. 1988, A\&A, 194, 125

van der Veen, W. E. C. J., \& Rugers, M. 1989, A\&A, 226, 183

Volk, K., \& Kwok, S. 1988, ApJ, 331, 435

Welty, A. D., Fix, J. D., \& Mutel, R. L. 1987, ApJ, 318, 852

Yates, J. A., \& Cohen, R. J. 1994, MNRAS, 270, 958

Zijlstra, A. A., Te Lintel Hekkert, P., Pottash, S. R., et al. 1989, A\&A, 217,157

Zijlstra, A. A., Chapman, J. M., Te Lintel Hekkert, P., et al. 2001, MNRAS, 322, 280 


\section{Online Material}




\section{Appendix A: Remarks on individual sources}

\section{A.1. "Mira-like"}

IRAS 15226-3603: We did not detect any emission from this source either at 1665 or at $1667 \mathrm{MHz}$ in March 1994 although Sivagnanam et al. (1990) clearly detected a signal at $1667 \mathrm{MHz}$ in July 1986 with an intensity of $0.46 \mathrm{Jy}$ for the strongest spectral profile component. At the time of the observations by Sivagnanam et al. this source was not detected at $1612 \mathrm{MHz}$, thus this Mira is certainly a type I.

IRAS 15262+0400: At the time of our observations this source showed an intensity at $1667 \mathrm{MHz}$ seven times greater than that observed by Sivagnanam et al. (1990) in July 1986. Nevertheless, the intensity ratio $I_{\text {red peak }} / I_{\text {blue peak }}$ (where "red peak" and "blue peak" stand for the red-shifted and blueshifted emission respectively) stayed the same. We detected faint non-polarized emission $(\simeq 0.2 \mathrm{~K})$ at $1665 \mathrm{MHz}$ internal in velocity to that observed at $1667 \mathrm{MHz}$ (where "internal (in velocity)" should be understood as defined in Sect. 3, meaning in this case that the signal is located between the blue and red peaks observed at $1667 \mathrm{MHz}$ ).

IRAS 20396-0826: This identified Mira, also known as XX Aqr, has an optical period of 334 days (Kholopov et al. $1985, G C V S)$. It was classified by Sivagnanam et al. (1990) as a "peculiar object" due to the difference between the spectral profile shape observed at 1612 and that observed at $1667 \mathrm{MHz}$. The $1612 \mathrm{MHz}$ emission shows a double-peaked profile covering a velocity range of $6 \mathrm{~km} \mathrm{~s}^{-1}$. The $1667 \mathrm{MHz}$ emission observed by Sivagnanam et al. is internal in velocity to that observed at $1612 \mathrm{MHz}$ and has a plateau-shaped profile. Our observations show that the $1665 \mathrm{MHz}$ emission is internal in velocity to that observed at $1667 \mathrm{MHz}$ with a single peak centred on the stellar velocity. A strong acceleration $(\epsilon>1)$ at the location of the $1665 \mathrm{MHz}$ emission could produce such a spectral profile shape (cf. Chapman \& Cohen 1985), strengthening the hypothesis of a velocity gradient in the $\mathrm{OH}$ shell of this source as proposed by Sivagnanam et al.

IRAS 20547+0247: This source, also known as U Equ, was indexed by Sivagnanam et al. as the Mira having the smallest expansion velocity with a value of $2.4 \mathrm{~km} \mathrm{~s}^{-1}$ (cf. their Table 1). The $1667 \mathrm{MHz}$ intensity of this source has substantially dropped. The blue-shifted peak which was already very faint at the time of the observations by Sivagnanam et al. in May 1987 is no longer detected in our observations. We detected a very faint $1665 \mathrm{MHz}$ signal only in the red-shifted peak centred at the same velocity as the emission we observed at $1667 \mathrm{MHz}$.

IRAS 22525+6033: Sivagnanam et al. classified this source as a "peculiar object" due to its spectral profile at $1667 \mathrm{MHz}$. Our observations indeed confirm the non-standard profile shape in both main lines, with multiple components quite well detached most of which are strongly right-hand polarized (cf. Fig. A.1 showing the Stokes $V$ spectra for both main lines).

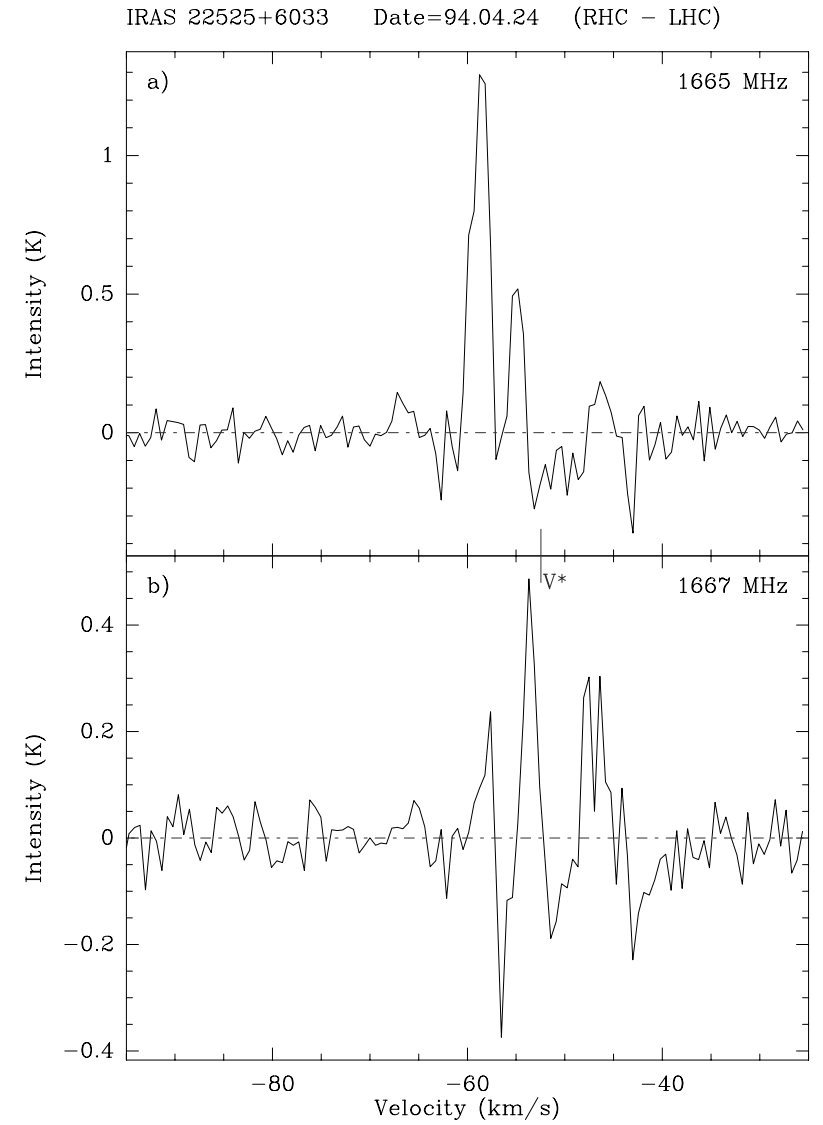

Fig. A.1. Stokes $V$ spectra of IRAS 22525+6033: a) at $1665 \mathrm{MHz}$; b) at $1667 \mathrm{MHz} . V_{*}$ is the stellar velocity inferred from the present work.

The resulting spectral profiles in the two circular polarizations are therefore very different (cf. Fig. B.1). Sivagnanam et al. inferred an expansion velocity of $4.4 \mathrm{~km} \mathrm{~s}^{-1}$. From our observations we infer an expansion velocity more than twice that value: $9-10 \mathrm{~km} \mathrm{~s}^{-1}$.

\section{A.2. $\mathrm{OH} / \mathrm{IR}$ stars from the LDSS region}

IRAS 17482-2824: Emission at 1667 and $1665 \mathrm{MHz}$ is present. The velocity location for the red- and blue-shifted peaks is about the same in both lines but the $1667-\mathrm{MHz}$ red-shifted peak emission overshoots that at $1665 \mathrm{MHz}$ by about $1 \mathrm{~km} \mathrm{~s}^{-1}$. Even though there is a separation of two years between the $1667-\mathrm{MHz}$ observations made by David et al. (1993a) and ours, there is no significant change in the spectral profile and intensity ratio $I_{\text {red peak }} / I_{\text {blue peak }}$.

IRAS 17505-3143: The comparison between the 1667-MHz observations made by David et al. (1993a) and ours showed a noticeable change in both the flux and the intensity ratio $I_{\text {red peak }} / I_{\text {blue peak }}$ between the two epochs of observations. We did not detect any $1665 \mathrm{MHz}$ emission. This source was observed repeatedly at $1612 \mathrm{MHz}$. In particular, Te Lintel et al. (1991) observed it in November 1985. They found an expansion velocity of $15 \mathrm{~km} \mathrm{~s}^{-1}$ with the blue- and red-shifted 
S. Etoka and A. M. Le Squeren: OH spectral evolution of oxygen-rich late-type stars, Online Material p 3

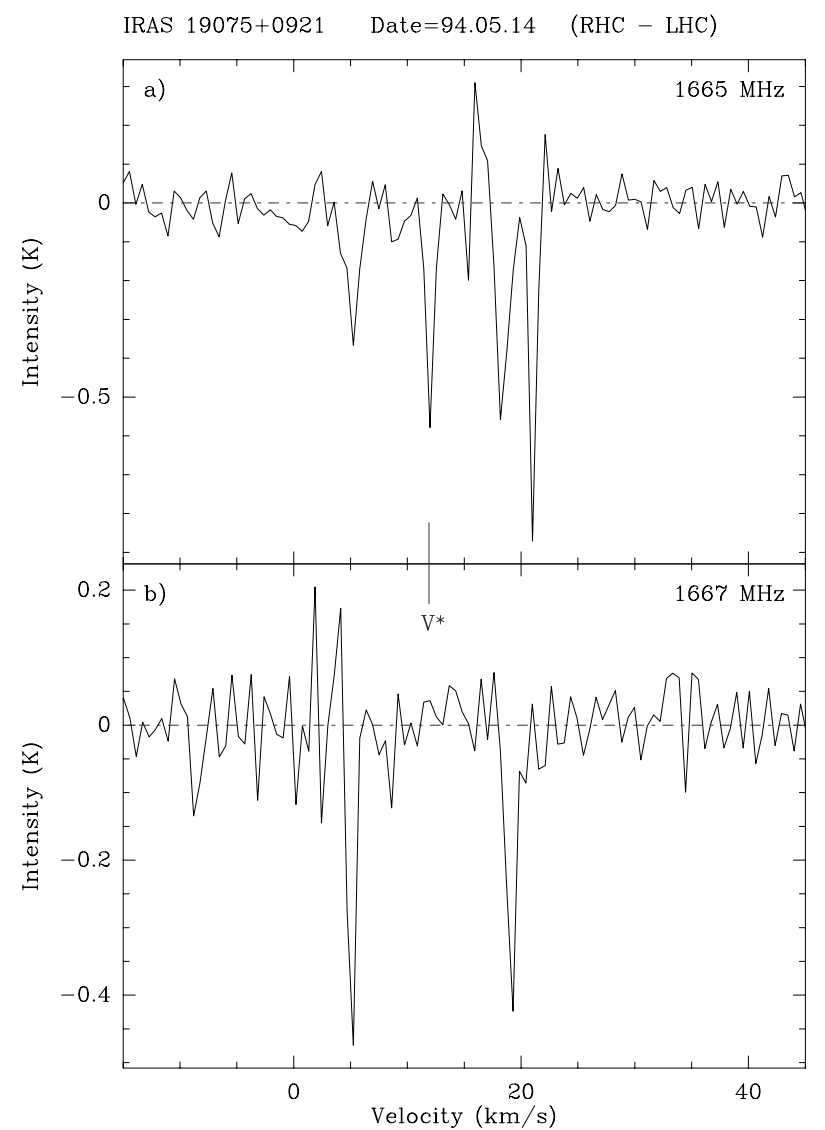

Fig. A.2. Stokes $V$ spectra of IRAS 19075+0921: a) at $1665 \mathrm{MHz}$; b) at $1667 \mathrm{MHz}$.

peaks located at -12.2 and $+19.2 \mathrm{~km} \mathrm{~s}^{-1}$ respectively (cf. their Table 2). From our $1667 \mathrm{MHz}$ observation we found the blueand red-shifted peaks to be centred at -12.9 and $+20.3 \mathrm{~km} \mathrm{~s}^{-1}$ respectively and an expansion velocity of $16.6 \mathrm{~km} \mathrm{~s}^{-1}$ in agreement with David et al. (1993a). This implies that the $1667 \mathrm{MHz}$ red-shifted peak overshoots that at $1612 \mathrm{MHz}$ by about $1 \mathrm{~km} \mathrm{~s}^{-1}$.

IRAS 19075+0921: The spectral profile of this source shows detached multi-components in both main lines leading to a non-standard shape, particularly noticeable at $1665 \mathrm{MHz}$. Like IRAS 22525+6033, this source shows a strong degree of polarization. Nevertheless, in this object the left-handed polarization prevails as can be seen in Fig. A.2 which displays the Stokes $V$ spectra in both main lines. As a result of the strong degree of circular polarization of some of the spectral components, the profile is quite different in both circular polarizations for both main lines. The $1667 \mathrm{MHz}$ spectrum profile obtained by Le Squeren et al. (1992) in January 1986 is similar to ours. The intensity ratio $I_{\text {red peak }} / I_{\text {blue peak }}$ did not show any significant change bewteen the two sets of observations. We also found the same characteristic velocities as those given by Le Squeren et al. (1992, their Table 1).

IRAS 19161+2343: We detected only a very faint and narrow emission in the red-shifted peak at $1665 \mathrm{MHz}$. The $1667 \mathrm{MHz}$ red-shifted peak overshoots that at $1665 \mathrm{MHz}$ by $\sim 1 \mathrm{~km} \mathrm{~s}^{-1}$.
No significant change in the profile shape can be seen between the observations made by David et al. (1993a) in May 1990 and ours.

IRAS 19190+1128: This source shows very faint and asymmetric emission at $1667 \mathrm{MHz}$. In particular, the blue-shifted peak hardly reached a $2 \sigma$ detection. We do not notice any significant change in the profile shape between the observations made by David et al. (1993a) in August 1986 and ours, performed 8 years later. We did not detect any emission at $1665 \mathrm{MHz}$.

IRAS 19244+1115: This source, also known as IRC 10420, has quite a broad spectral profile. Because of the protocol of our observations the red-shifted part of the spectra has been cut. Indeed, if we refer to the observations made by David et al. (1993a) at $1667 \mathrm{MHz}$, we can clearly see the existence of a strong red-shifted component centred at $+110 \mathrm{~km} \mathrm{~s}^{-1}$, outside our bandwidth coverage. Although it was covered by our bandwidth, we did not detect the most blue-shifted component centred at $+41 \mathrm{~km} \mathrm{~s}^{-1}$ seen in the profile published by David et al. That is most probably due to the "band edge effects" concerning the first and last 5 to 10 channels of the band. Due to the inadequate protocol of observation for this particular source, the stellar and expansion velocities given in Tables C.1 and C.2 should be treated with caution. This source was not used in the statistical study on the expansion velocity presented in Sect. 4.4.

\section{A.3. $\mathrm{OH} / \mathrm{IR}$ stars from the $\mathrm{R}_{2}$ region}

IRAS 17220-2448: We did not detect any emission at $1667 \mathrm{MHz}$, but a faint, narrow left-hand polarized component centred at $V=+45.3 \mathrm{~km} \mathrm{~s}^{-1}$ was detected at $1665 \mathrm{MHz}$. Considering the velocity characteristics given by David et al. (1993a,$V_{*}=+39.4 \mathrm{~km} \mathrm{~s}^{-1}$ and $V_{\exp }=15.4 \mathrm{~km} \mathrm{~s}^{-1}$ ) this component should be an $\mathrm{OH}$ maser signature of an inner region belonging to the back part of the shell.

IRAS 17317-3331: The $1667 \mathrm{MHz}$ emission of this source exhibits an inter-peak signal. At $1665 \mathrm{MHz}$ we observed an internal group of components between the two asymmetrical red-and blue-shifted peaks. This internal peak is centred about the stellar velocity and its intensity is of the same order as that observed for the blue-shifted peak. This source was also observed at $1612 \mathrm{MHz}$ by Te Lintel et al. (1991) and by David et al. (1993a). From our observations in the main lines, we found the same stellar velocity as David et al. which is in agreement with that given by Te Lintel et al. considering their spectral resolution. At $1612 \mathrm{MHz}$ the spectral profile does not have any noticeable particularity. Both Te Lintel et al. and David et al. classified it in the "standard double-peaked profile" category.

IRAS 17385-3332: Both main lines exhibit faint emission. The blue-shifted peak is not detected at $1667 \mathrm{MHz}$ and only just detected at $1665 \mathrm{MHz}$. At $1665 \mathrm{MHz}$, we clearly observe 
S. Etoka and A. M. Le Squeren: $\mathrm{OH}$ spectral evolution of oxygen-rich late-type stars, Online Material p 4

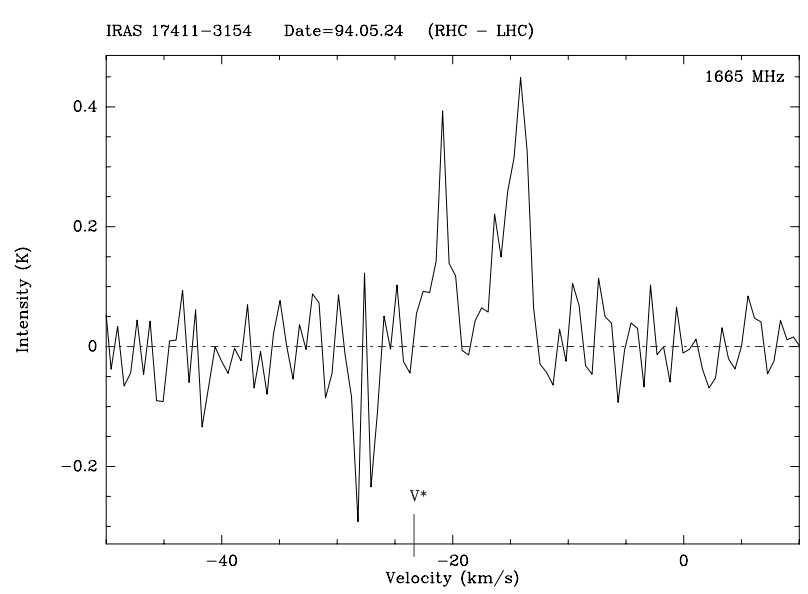

Fig. A.3. Stokes $V$ spectrum of IRAS 17411-3154 at $1665 \mathrm{MHz}$.

an internal peak centred on the stellar velocity, and the bluest narrow component we detected is centred at the same velocity as the $1612 \mathrm{MHz}$ blue-shifted peak as given by Te Lintel et al. (1991) and David et al. (1993b).

IRAS 17392-3319: We did not detect any emission at $1665 \mathrm{MHz}$. The $1667 \mathrm{MHz}$ emission shows a standard double-peaked profile. The profile and intensity have not shown any noticeable change between our observations carried out in 1994 and those performed by David et al. 8 years earlier.

IRAS 17411-3154: The $1665 \mathrm{MHz}$ emission exhibits a non-standard profile shape where at least 8 well-defined components are observed, of which 4 are coming from strong internal emission. Interestingly, the back part of the shell (i.e., $V>V_{*}$ ) shows quite strong right-handed polarization while the front part (i.e., $V<V_{*}$ ) is left-hand polarized (Fig. A.3). A similar phenomenon has already been observed in other sources (i.e., Ukita \& Le Squeren 1984; Szymczak et al. 2001), and can be explained by the Cook mechanism when the magnetic field is aligned on the line of sight (Cook 1966, 1977). The $1667 \mathrm{MHz}$ spectral profile is more typical although showing inter-peak emission. This source also exhibits strong $1612 \mathrm{MHz}$ emission with intensity reaching a value as high as 200-300 Jy (cf. Table 2 of Te Lintel et al. 1991 and Table 1 of David et al. 1993b). The expansion velocity inferred at $1612 \mathrm{MHz}$ is of the same order as that inferred at $1667 \mathrm{MHz}$ (i.e., $18.5 \mathrm{~km} \mathrm{~s}^{-1}$, Table C.2). On the other hand, the most red-shifted part of the $1665 \mathrm{MHz}$ emission was not detected, leading to a much smaller expansion velocity in that line.

IRAS 17418-2713: This source shows a single component at $1665 \mathrm{MHz}$ centred about $V=-21 \mathrm{~km} \mathrm{~s}^{-1}$, that is about the mid-point between the stellar velocity and the $1667 \mathrm{MHz}$ blue peak velocity. The expansion velocity inferred at $1667 \mathrm{MHz}$ is more than $1 \mathrm{~km} \mathrm{~s}^{-1}$ greater than that inferred by David et al. (1993b) and Te Lintel et al. (1991) from the $1612 \mathrm{MHz}$ emission. This is due to an overshoot of the $1667 \mathrm{MHz}$ blue peak over that at $1612 \mathrm{MHz}$.
IRAS 17550-2120: Emission is observed in both main lines but with an inverse intensity ratio $I_{\text {red peak }} / I_{\text {blue peak. The }}$ $1667 \mathrm{MHz}$ profile underwent a strong change of shape since 1987 when a triple-peaked profile shape was observed (cf. Fig. 2 of David et al. 1993a) while we detected only the two standard peaks. The extra internal component centred about $V=-17 \mathrm{~km} \mathrm{~s}^{-1}$ detected by David et al. vanished between the two epochs of observation. The nature of this event is unclear since the source has not been monitored.

IRAS 18033-2111: It seems that the $\mathrm{OH}$ emission reported by David et al. (1993a, their Fig. 2 and Table 1) corresponds to two distinct sources: one emitting in the velocity range [ -130 ; $-100 \mathrm{~km} \mathrm{~s}^{-1}$ (the one we observed) and the second emitting in the velocity range $[-70 ;-30] \mathrm{km} \mathrm{s}^{-1}$. The $1612 \mathrm{MHz}$ observations reported by Te Lintel et al. (1991) and David et al. (1993b) deal with the velocity range $[-70 ;-30] \mathrm{km} \mathrm{s}^{-1}$. To our knowledge no observation has been done at $1612 \mathrm{MHz}$ in the velocity range $[-130 ;-100] \mathrm{km} \mathrm{s}^{-1}$. At the date of our observation neither $1667 \mathrm{MHz}$ nor $1665 \mathrm{MHz}$ emissions were detected while David et al. (1993a) clearly reported emission at $1667 \mathrm{MHz}$. Thus, the source corresponding to the velocity range $[-130 ;-100] \mathrm{km} \mathrm{s}^{-1}$ is clearly variable.

IRAS 18135-1456: The $1667 \mathrm{MHz}$ spectrum of this source shows an asymmetric double-peaked profile. The $1665 \mathrm{MHz}$ spectrum is internal to that at $1667 \mathrm{MHz}$ and emission is observed over the whole velocity range (leading to its classification as a plateau-shaped profile). Actually, the two external components (i.e., the bluest and reddest Doppler shifted) are those with the faintest emission. In particular, if one excepts the strong component centred at $V=-9.4 \mathrm{~km} \mathrm{~s}^{-1}$, the general shape of the profile resembles a Gaussian. This could be the signature of an acceleration zone (cf. Chapman \& Cohen 1985).

\section{IRAS 18198-1249, IRAS 18257-1000,}

IRAS 18488-0107 and IRAS 19065+0832: These four sources were observed by Dickinson \& Turner (1991) in both main lines. All four sources show a standard double-peaked profile at $1667 \mathrm{MHz}$. We did not notice any significant change in their spectral profiles. We did not detect any $1665 \mathrm{MHz}$ either for IRAS 18198-1249 or for IRAS 18488-0107. We positively detected IRAS $18257-1000$ and IRAS $19065+0832$ at 1665 MHz. Dickinson \& Turner classified IRAS 18198-1249, IRAS 18257-1000 and IRAS 18488-0107 as sources for which the $1667 \mathrm{MHz}$ emission overshoots that at $1612 \mathrm{MHz}$.

IRAS 18348-0526: This source shows a strong inter-peak emission at $1667 \mathrm{MHz}$. At $1665 \mathrm{MHz}$ we also noted a non-zero inter-peak emission whose shape resembles a very flat-topped Gaussian centred on the stellar velocity. This kind of shape is similar to that presented by Bowers (1991) for an isotropic outflow in a spherical circumstellar shell with significant rotation. This hypothesis would imply a non-negligible rotation component in the external regions of the circumstellar shell considering the radius expected for the $\mathrm{OH}$ formation (Goldreich \& Scoville 1976; Huggins \& Glassgold 1982). This 
is very unlikely. Another more credible hypothesis regards the emission as the sum of two components. The first component, coming from an external $\mathrm{OH}$ region expanding radially with an acceleration such as $1>\epsilon>0$, would give the standard redand blue-shifted peaks with possibly a faint inter-peak signal. The second component would come from an inner $\mathrm{OH}$ strongly accelerating zone with $\epsilon>1$, given the internal Gaussian-like emission observed (cf. Chapman \& Cohen 1985, their Fig. 3). This scenario is sustained by the $1667 \mathrm{MHz}$ profile shape which also shows an inter-peak signal, strengthening the hypothesis that some acceleration is still present at the location of the main-line maser emission.

\section{IRAS 18432-0149, IRAS 18460-0254 and}

IRAS 19254+1631: The first two sources show faint emission at $1665 \mathrm{MHz}$ barely above the noise threshold. The $1667 \mathrm{MHz}$ spectra for all three sources did not show any significant change between the observations carried out by David et al. (1993a) and ours.
IRAS 19352+2030: At $1667 \mathrm{MHz}$ we detected emission from three peaks centred at $V=-8.70,-1.4$ and $+7.0 \mathrm{~km} \mathrm{~s}^{-1}$. The two most red- and blue-shifted peaks are very faint. Only the central peak at $V=-1.40 \mathrm{~km} \mathrm{~s}^{-1}$ was detected by David et al. (1993a). We also detected emission at $1665 \mathrm{MHz}$ centred at $V=-1.40 \mathrm{~km} \mathrm{~s}^{-1}$. This peak is significantly left-hand polarized (i.e., 15-20\%) in both main lines. This source was also observed at $1612 \mathrm{MHz}$ by David et al. (1993b). They only detected a single peak centred at $V=-3.22 \mathrm{~km} \mathrm{~s}^{-1}$.

IRAS 22177+5936: At $1667 \mathrm{MHz}$ this source shows a doublepeaked profile with strong emission between the the red-and blue-shifted peaks. At $1665 \mathrm{MHz}$ only the blue-shifted peak is clearly detected. The red-shifted peak is barely above the noise threshold in right-handed polarization. The spectral profiles obtained by Dickinson \& Turner (1991) are similar to ours for both main lines. This source was also observed at $1612 \mathrm{MHz}$ by Te Lintel et al. (1991). The characteristic velocities they found are in agreement with ours. 
S. Etoka and A. M. Le Squeren: OH spectral evolution of oxygen-rich late-type stars, Online Material p 6

\section{Appendix B: Spectral line atlas}
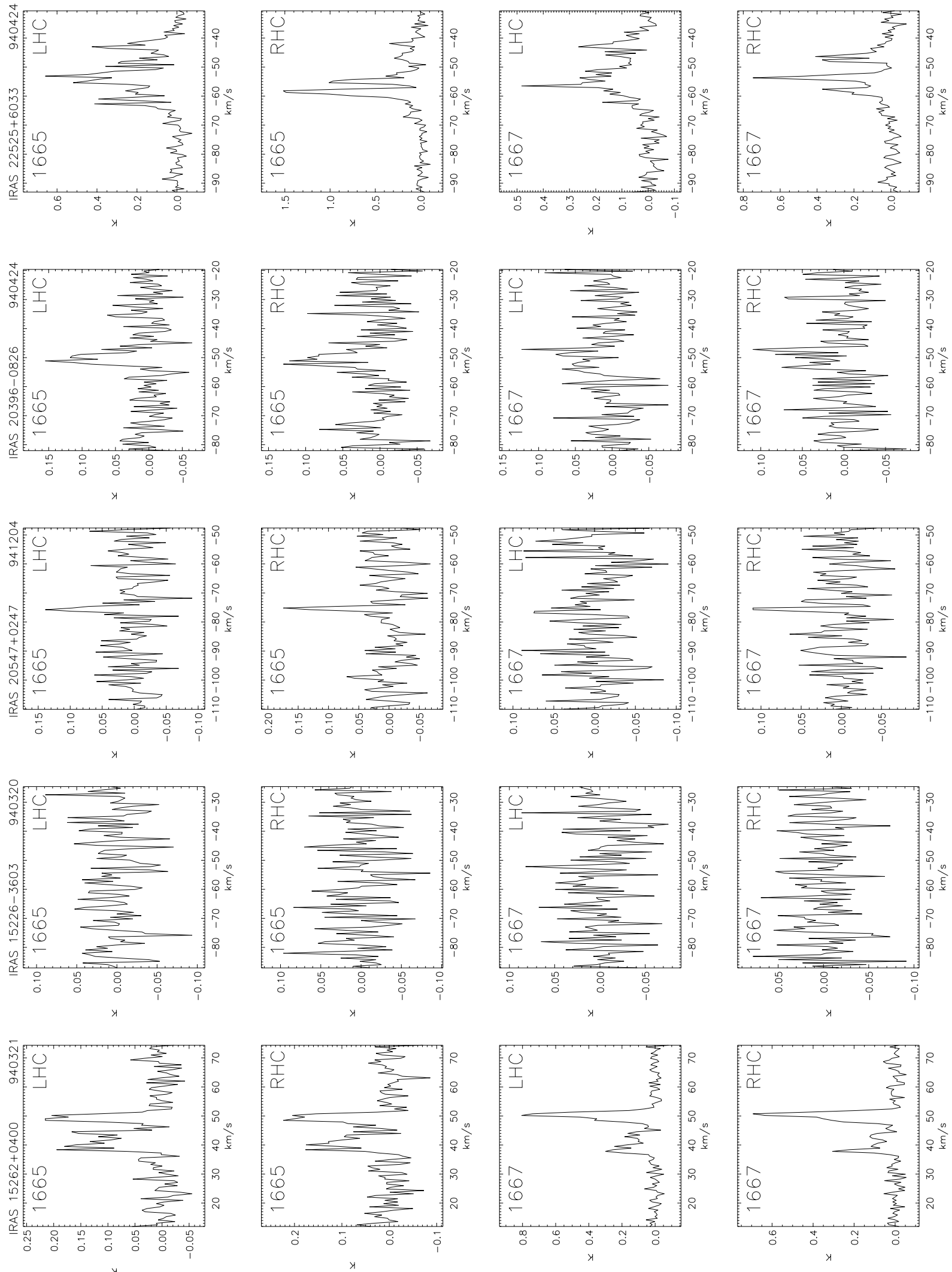

Fig. B.1. Spectra of the sources at 1665 and $1667 \mathrm{MHz}$ in the left- (LHC) and right-handed (RHC) polarizations, appearing in increasing value of the [25-12] colour index. 
S. Etoka and A. M. Le Squeren: OH spectral evolution of oxygen-rich late-type stars, Online Material p 7
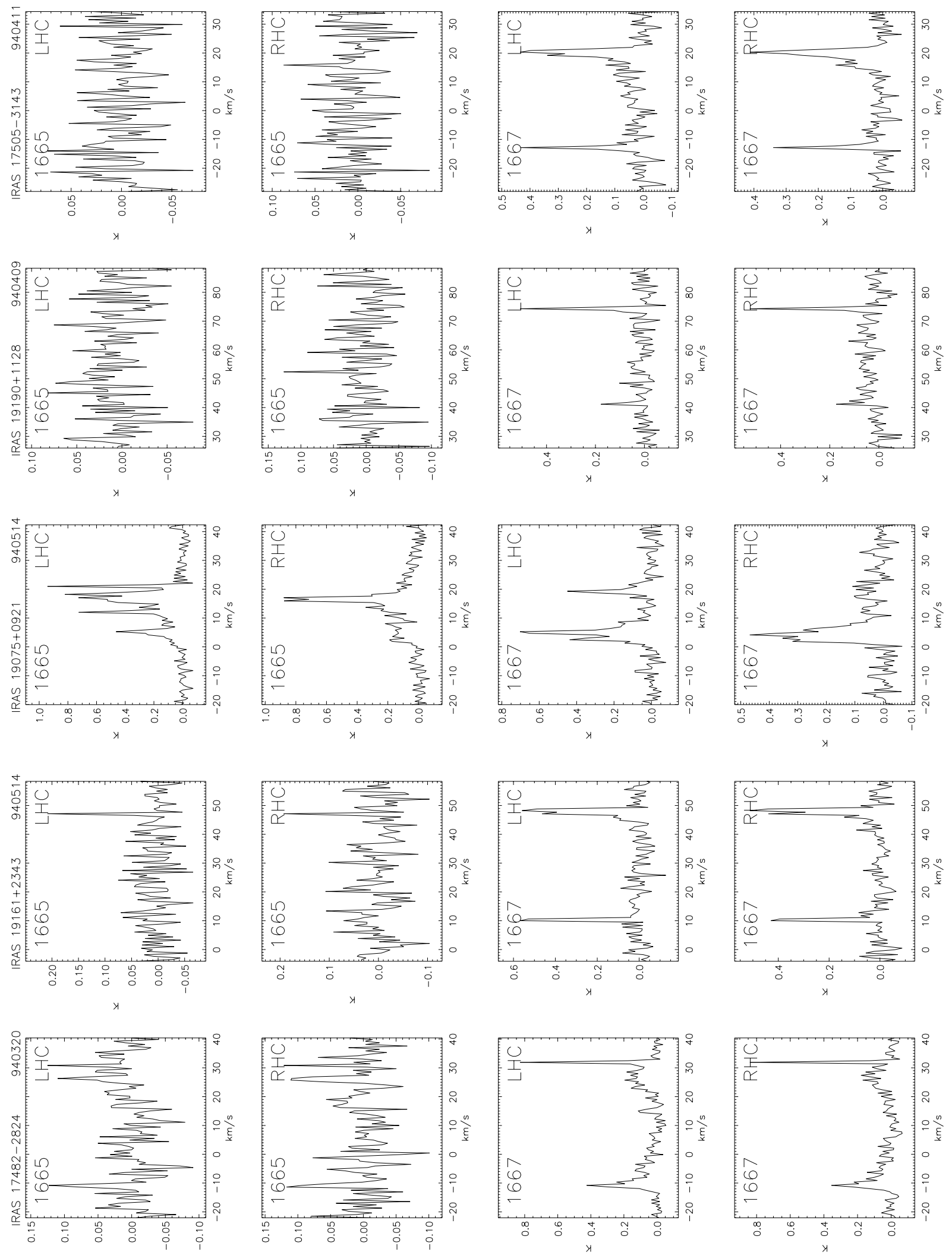

Fig. B.1. continued. 
S. Etoka and A. M. Le Squeren: $\mathrm{OH}$ spectral evolution of oxygen-rich late-type stars, Online Material $p 8$
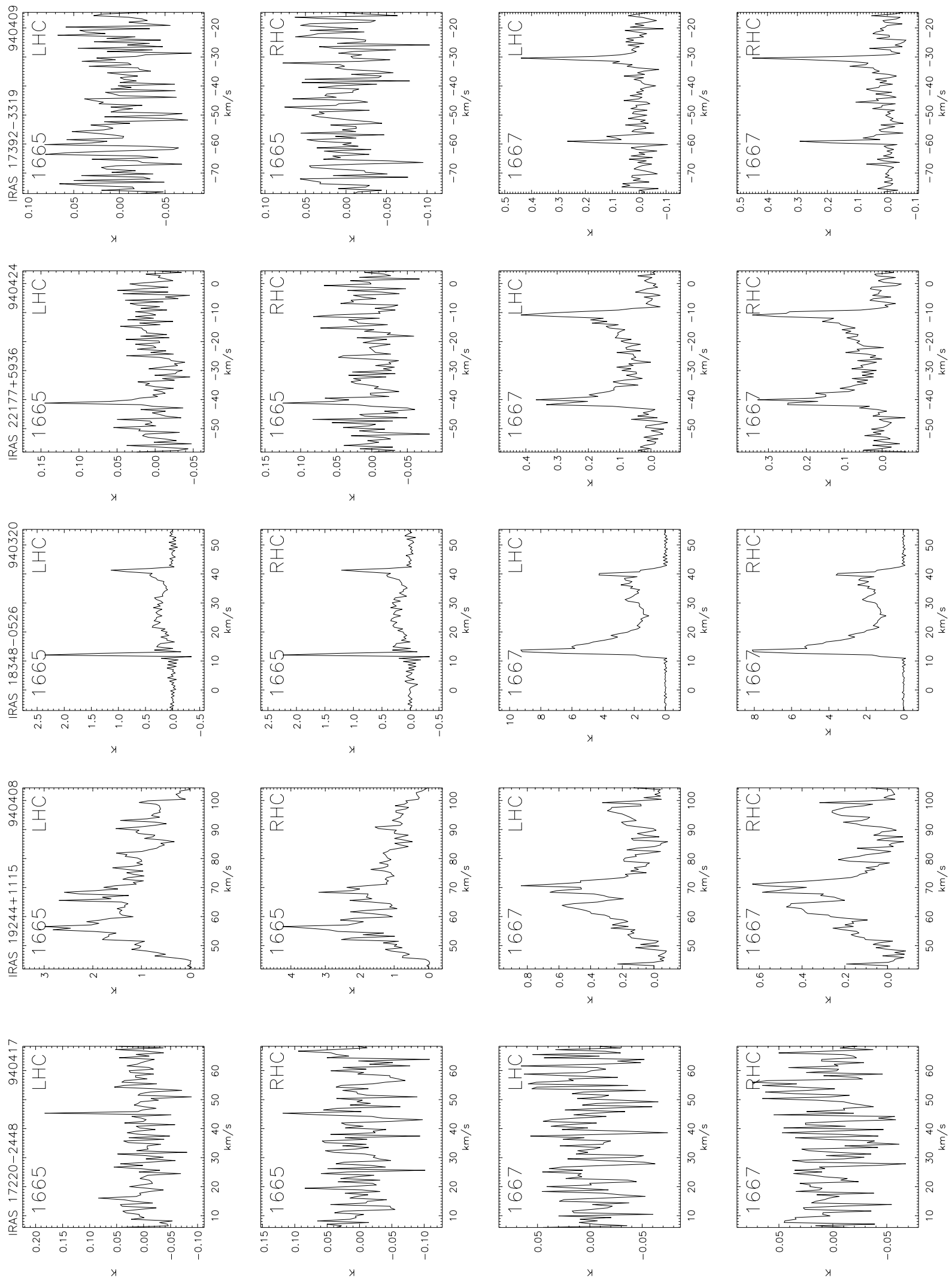

Fig. B.1. continued. 
S. Etoka and A. M. Le Squeren: OH spectral evolution of oxygen-rich late-type stars, Online Material p 9
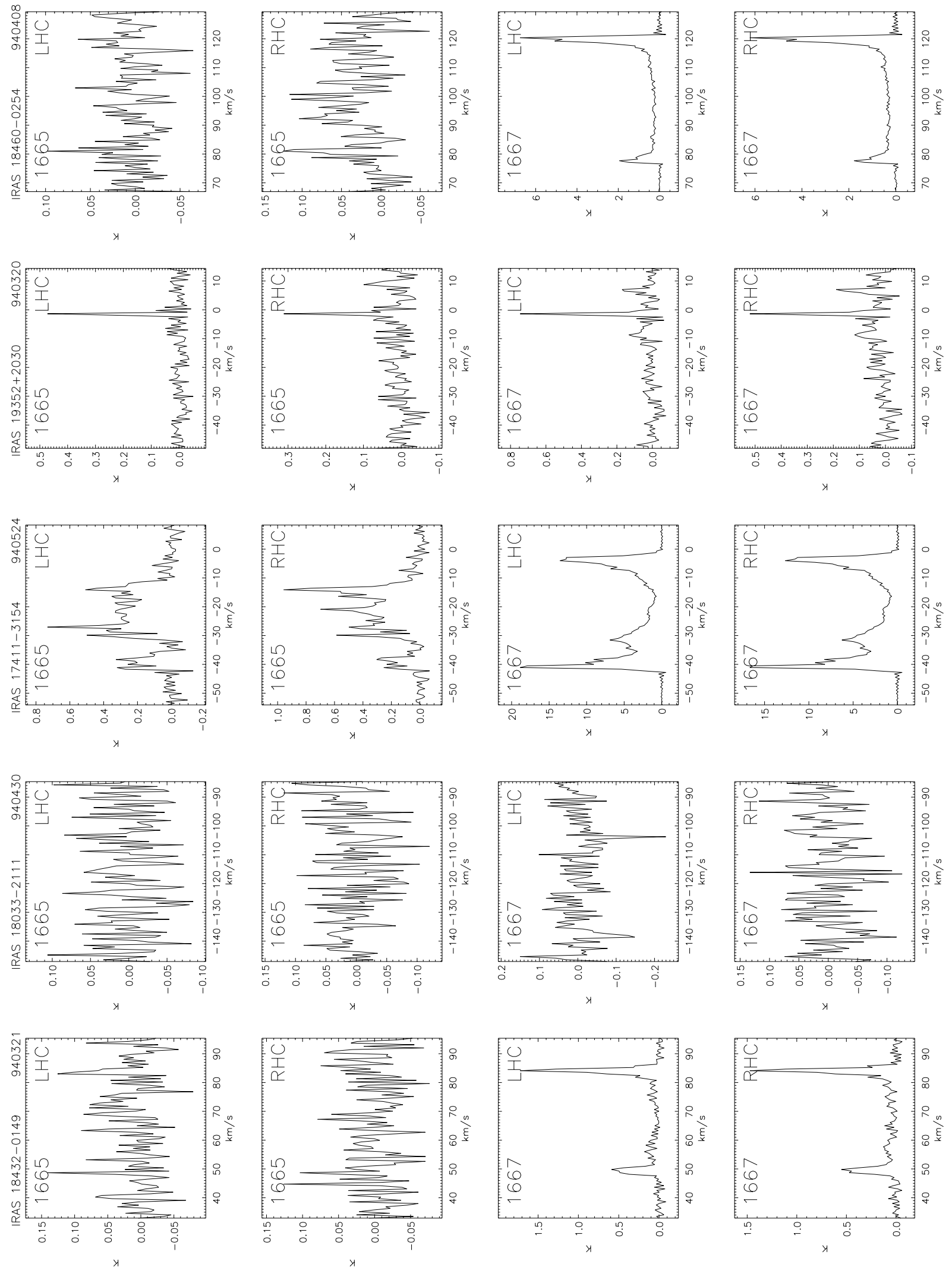

Fig. B.1. continued. 
S. Etoka and A. M. Le Squeren: OH spectral evolution of oxygen-rich late-type stars, Online Material p 10
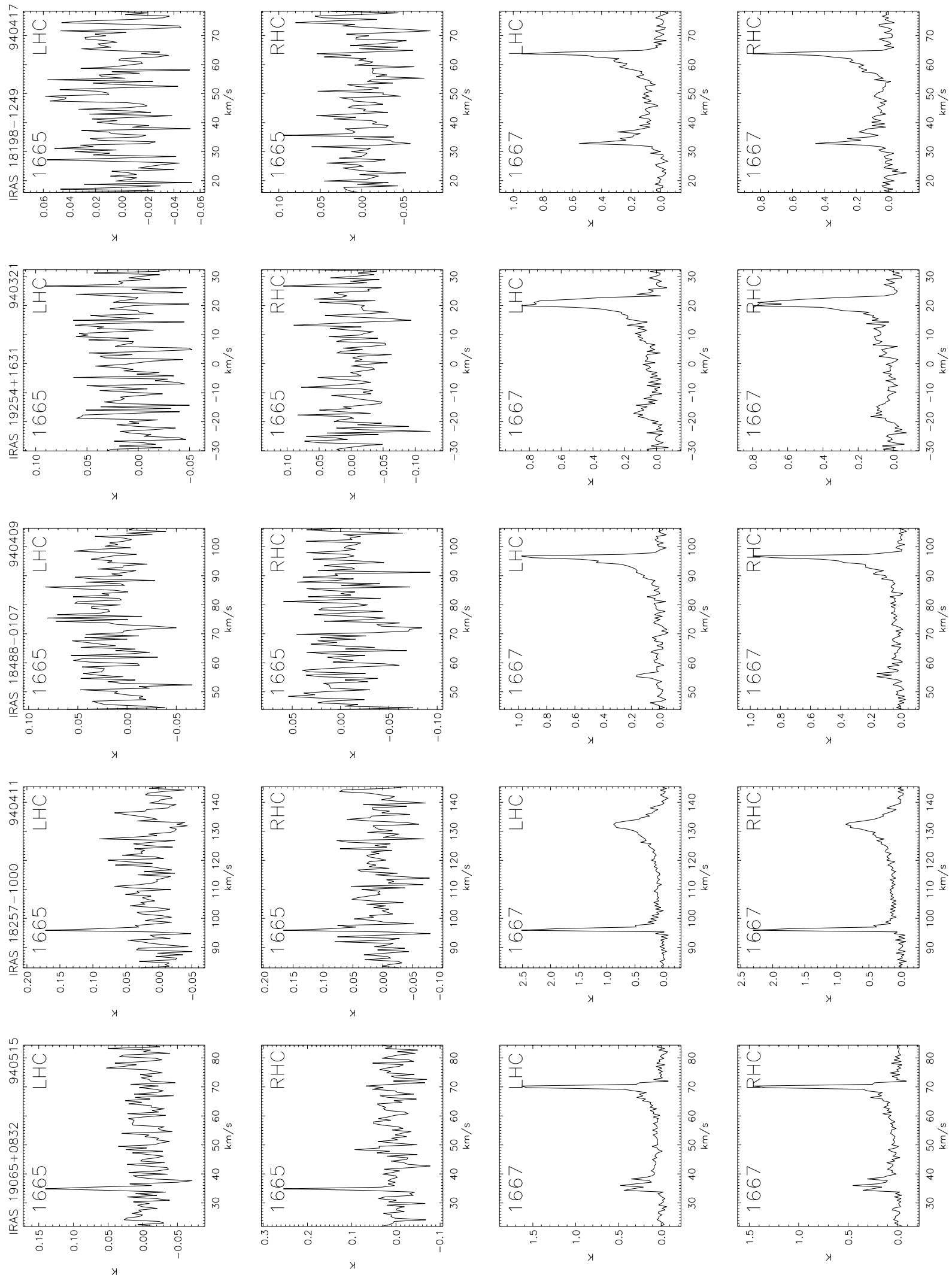

Fig. B.1. continued. 
S. Etoka and A. M. Le Squeren: $\mathrm{OH}$ spectral evolution of oxygen-rich late-type stars, Online Material p 11
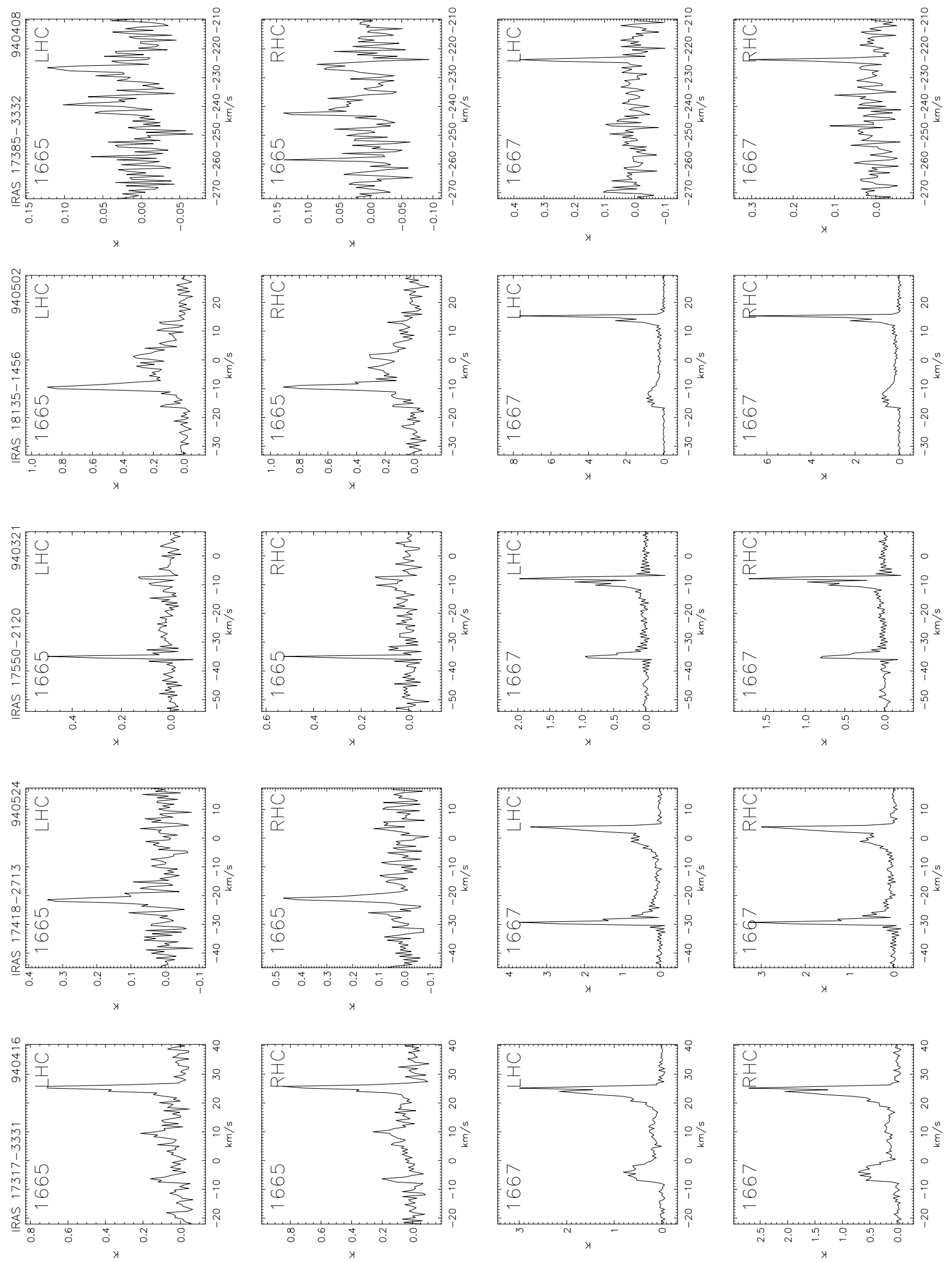

Fig. B.1. continued. 


\section{Appendix C: Spectral measurement results}

Table C.1. Results of the spectral measurements at $1665 \mathrm{MHz}$.

\begin{tabular}{|c|c|c|c|c|c|c|c|c|c|c|c|c|c|c|c|}
\hline \multirow[t]{2}{*}{ Iras name } & \multirow[t]{2}{*}{$\mathrm{ps}^{2}$} & \multicolumn{2}{|c|}{$\begin{array}{c}V_{b}^{1} \\
\mathrm{~km} \mathrm{~s}^{-1}\end{array}$} & \multicolumn{2}{|c|}{$\begin{array}{c}V_{r}^{1} \\
\mathrm{~km} \mathrm{~s}^{-1}\end{array}$} & \multicolumn{2}{|c|}{$\begin{array}{c}V_{\text {int }}^{1,3} \\
\mathrm{~km} \mathrm{~s}^{-1}\end{array}$} & \multirow[t]{2}{*}{$\begin{array}{r}V_{*}^{1} \\
\mathrm{~km} \mathrm{~s}^{-1}\end{array}$} & \multirow[t]{2}{*}{$\begin{array}{r}V_{\exp }^{1} \\
\mathrm{~km} \mathrm{~s}^{-1}\end{array}$} & \multicolumn{2}{|c|}{$\begin{array}{c}S_{b}^{1} \\
\mathrm{Jy}\end{array}$} & \multicolumn{2}{|c|}{$\begin{array}{l}S_{r}^{1} \\
\mathrm{Jy}\end{array}$} & \multicolumn{2}{|c|}{$\begin{array}{c}S_{\text {int }}^{1,3} \\
\text { Jy }\end{array}$} \\
\hline & & $\mathrm{L}^{1}$ & $\mathrm{R}^{1}$ & $\mathrm{~L}$ & $\mathrm{R}$ & $\mathrm{L}$ & $\mathrm{R}$ & & & $\mathrm{L}$ & $\mathrm{R}$ & $\mathrm{L}$ & $\mathrm{R}$ & $\mathrm{L}$ & $\mathrm{R}$ \\
\hline $15226-3603$ & & $\ldots$ & $\ldots$ & $\ldots$ & $\ldots$ & & & & & $\ldots$ & $\ldots$ & $\ldots$ & $\ldots$ & & \\
\hline $15262+0400$ & & 38.38 & 38.38 & 49.06 & 48.50 & & & 43.6 & 5.2 & 0.19 & 0.18 & 0.22 & 0.22 & & \\
\hline 20396-0826 & c?p? & $\ldots$ & $\ldots$ & & $\ldots$ & -51.13 & -52.25 & & & $\ldots$ & $\ldots$ & $\ldots$ & $\ldots$ & 0.16 & 0.13 \\
\hline $20547+0247$ & & $\ldots$ & $\ldots$ & -75.75 & -75.19 & & & & & $\ldots$ & $\ldots$ & 0.14 & 0.17 & & \\
\hline $22525+6033$ & $\mathrm{p}$ & & $-67.19 ?$ & & & -55.38 & -55.38 & & & & $0.19 ?$ & & & 0.56 & 1.01 \\
\hline & & -62.69 & & -41.87 & -41.87 & -53.12 & -53.12 & -52.3 & 10.4 & 0.41 & & 0.25 & 0.34 & 0.66 & 0.38 \\
\hline & & -61.00 & & -43.00 & & -49.75 & & & & 0.39 & & 0.43 & & 0.36 & \\
\hline & & -58.19 & -58.19 & & -46.94 & -48.62 & & & & 0.26 & 1.52 & & 0.29 & 0.29 & \\
\hline $17482-2824$ & & 10.81 & -11.38 & 30.81 & 30.81 & & & 9.9 & 20.9 & 0.12 & 0.12 & 0.13 & 0.12 & & \\
\hline $17505-3143$ & & $\ldots$ & $\ldots$ & $\ldots$ & $\ldots$ & & & & & $\ldots$ & $\ldots$ & $\ldots$ & $\ldots$ & & \\
\hline $19075+0921$ & $\mathrm{p}$ & 5.25 & 2.44 & 21.00 & 17.06 & 12.00 & 8.06 & 11.4 & 7.6 & 0.46 & 0.19 & 0.94 & 0.87 & 0.72 & 0.22 \\
\hline & & $\ldots$ & $\ldots$ & $\ldots$ & $\ldots$ & 17.06 & 10.88 & & & $\ldots$ & $\ldots$ & $\ldots$ & $\ldots$ & 0.73 & 0.22 \\
\hline & & $\cdots$ & $\ldots$ & $\ldots$ & $\ldots$ & 18.19 & 13.69 & & & $\ldots$ & $\ldots$ & $\ldots$ & $\ldots$ & 0.82 & 0.34 \\
\hline $19161+2343$ & & $\ldots$ & $\ldots$ & 47.13 & 47.13 & & & & & $\ldots$ & $\ldots$ & 0.21 & 0.19 & & \\
\hline $19190+1128$ & & $\ldots$ & $\ldots$ & $\ldots$ & $\ldots$ & & & & & $\ldots$ & $\ldots$ & $\ldots$ & $\ldots$ & & \\
\hline 19244+1115 & $\mathrm{p}$ & 56.56 & 56.56 & 99.31 & 98.13 & 65.56 & 58.81 & 77.6 & 21.1 & 2.99 & 4.23 & 1.05 & 0.97 & 2.69 & 2.56 \\
\hline & & $\ldots$ & $\ldots$ & $\ldots$ & $\ldots$ & 68.38 & 61.62 & & & $\ldots$ & $\ldots$ & $\ldots$ & $\ldots$ & 2.59 & 2.27 \\
\hline & & $\cdots$ & $\ldots$ & ... & $\ldots$ & 76.81 & 65.56 & & & $\ldots$ & $\ldots$ & $\ldots$ & $\ldots$ & 1.60 & 2.29 \\
\hline & & $\ldots$ & $\ldots$ & ... & $\ldots$ & 81.88 & 68.38 & & & $\ldots$ & $\ldots$ & $\ldots$ & $\ldots$ & 1.51 & 3.19 \\
\hline & & $\ldots$ & $\ldots$ & $\ldots$ & $\ldots$ & 90.31 & 76.25 & & & $\ldots$ & $\ldots$ & $\ldots$ & $\ldots$ & 1.53 & 1.68 \\
\hline & & $\ldots$ & $\ldots$ & ... & $\ldots$ & 93.13 & 81.88 & & & $\ldots$ & $\ldots$ & $\ldots$ & $\ldots$ & 1.43 & 1.32 \\
\hline & & $\ldots$ & $\ldots$ & $\ldots$ & $\ldots$ & & 90.88 & & & $\ldots$ & $\ldots$ & $\ldots$ & $\ldots$ & & 1.56 \\
\hline & & $\ldots$ & $\ldots$ & $\ldots$ & $\ldots$ & & 93.13 & & & $\ldots$ & $\ldots$ & $\ldots$ & $\ldots$ & & 1.06 \\
\hline & & $\ldots$ & $\ldots$ & ... & $\ldots$ & & 96.50 & & & $\ldots$ & $\ldots$ & $\ldots$ & $\ldots$ & & 0.95 \\
\hline 17317-3331 & $\mathrm{ci}$ & -6.31 & -6.31 & 25.19 & 25.75 & 9.44 & 10.00 & 9.6 & 15.9 & 0.16 & 0.20 & 0.71 & 0.85 & 0.21 & 0.26 \\
\hline $17220-2448$ & & $\ldots$ & $\ldots$ & 45.31 & 45.31 & & & & & $\ldots$ & $\ldots$ & 0.18 & 0.12 & & \\
\hline $17385-3332$ & $\mathrm{ci}$ & $-\ldots$ & -258.25 & -226.50 & -225.37 & -239.44 & -242.15 & -241.8 & 16.2 & $0.06 ?$ & 0.14 & 0.12 & 0.09 & 0.10 & 0.14 \\
\hline 17392-3319 & & $-60.19 ?$ & $\ldots$ & $-22.50 ?$ & $\ldots$ & & & $-41.3 ?$ & $18.8 ?$ & $0.08 ?$ & $\ldots$ & $0.07 ?$ & $\ldots$ & & \\
\hline 17411-3154 & $\mathrm{p}$ & -38.31 & -38.31 & -14.13 & -14.13 & -27.06 & -20.88 & -26.2 & 12.1 & 0.32 & 0.59 & 0.51 & 0.96 & 0.73 & 0.59 \\
\hline $17418-2713$ & $\mathrm{ci}$ & $\ldots$ & $\ldots$ & $\ldots$ & $\ldots$ & -21.44 & -20.88 & & & $\ldots$ & $\ldots$ & $\ldots$ & $\ldots$ & 0.34 & 0.47 \\
\hline $17550-2120$ & & -34.94 & -34.94 & -7.37 & -7.37 & & & -21.1 & 13.8 & 0.50 & 0.53 & 0.13 & 0.14 & & \\
\hline 18033-2111 & & & $\ldots$ & & $\ldots$ & & & & & $\ldots$ & $\ldots$ & $\ldots$ & $\ldots$ & & \\
\hline 18135-1456 & $\mathrm{p}$ & -16.19 & -16.19 & 13.06 & 13.06 & -9.44 & -9.44 & -1.6 & 14.6 & 0.16 & 0.15 & 0.16 & 0.18 & 0.90 & 0.91 \\
\hline & & $\ldots$ & $\ldots$ & $\ldots$ & $\ldots$ & -2.13 & -2.67 & & & $\ldots$ & $\ldots$ & $\ldots$ & $\ldots$ & 0.31 & 0.32 \\
\hline & & $\ldots$ & $\ldots$ & $\ldots$ & $\ldots$ & 1.25 & 1.81 & & & $\ldots$ & $\ldots$ & $\ldots$ & $\ldots$ & 0.33 & 0.31 \\
\hline & & $\ldots$ & $\ldots$ & $\ldots$ & $\ldots$ & 5.75 & 5.75 & & & $\ldots$ & $\ldots$ & $\ldots$ & $\ldots$ & 0.16 & 0.14 \\
\hline & & $\ldots$ & $\ldots$ & $\ldots$ & $\ldots$ & 10.25 & & & & $\ldots$ & $\ldots$ & $\ldots$ & $\ldots$ & 0.18 & \\
\hline $18198-1249$ & & $\ldots$ & $\ldots$ & ... & $\ldots$ & & & & & $\ldots$ & $\ldots$ & $\ldots$ & $\ldots$ & & \\
\hline $18257-1000$ & & 95.87 & 95.87 & $\ldots$ & $\ldots$ & & & & & 0.17 & 0.17 & $\ldots$ & $\ldots$ & & \\
\hline $18348-0526$ & $\mathrm{~s}$ & 12.06 & 12.06 & 41.31 & 41.31 & 26.69 & 26.13 & 26.7 & 14.6 & 2.36 & 2.24 & 1.14 & 1.21 & 0.31 & 0.30 \\
\hline $18432-0149$ & & 48.69 & $\ldots$ & 83.00 & $\ldots$ & & & 65.8 & 17.2 & 0.14 & $\ldots$ & 0.13 & $\ldots$ & & \\
\hline $18460-0254$ & s? & 81.00 & 81.00 & $\ldots$ & $\ldots$ & & $100.69 ?$ & & & 0.10 & 0.12 & $\ldots$ & $\ldots$ & & $0.12 ?$ \\
\hline 18488-0107 & & $\ldots$ & $\ldots$ & ... & $\ldots$ & & & & & $\ldots$ & $\ldots$ & $\ldots$ & $\ldots$ & & \\
\hline $19065+0832$ & & 34.87 & 34.87 & ... & $\ldots$ & & & & & 0.14 & 0.25 & $\ldots$ & $\ldots$ & & \\
\hline 19254+1631 & & $\ldots$ & $\ldots$ & $\ldots$ & $\ldots$ & & & & & $\ldots$ & $\ldots$ & $\ldots$ & $\ldots$ & & \\
\hline $19352+2030$ & ci & $\ldots$ & $\ldots$ & $\ldots$ & $8.75 ?$ & -1.37 & -1.37 & & & $\ldots$ & $\ldots$ & $\ldots$ & $0.10 ?$ & 0.47 & 0.31 \\
\hline $22177+5936$ & & -41.19 & -41.19 & $\ldots$ & $-11.37 ?$ & & & $-26.3 ?$ & $14.9 ?$ & 0.14 & 0.13 & ... & $0.08 ?$ & & \\
\hline
\end{tabular}

(1) L: left-handed polarization; R: right-handed polarization;

(2) profile shape characteristic:

p: plateau profile; c: profile with only a single peak centred on the stellar velocity; s: "standard" profile with inter-peak signal; ci: profile with an internal component or group of components (well detached from the two standard peaks);

(3) $V_{\text {int }}$ : in one of the mentioned cases. When the profile is triple-peaked, the velocity of the component showing the strongest intensity (the latter might not be centred on the stellar velocity) is at least given. $S_{\text {int }}$ : intensity corresponding to $V_{\text {int }}$. 
S. Etoka and A. M. Le Squeren: $\mathrm{OH}$ spectral evolution of oxygen-rich late-type stars, Online Material p 13

Table C.2. Results of the spectral measurements at $1667 \mathrm{MHz}$.

\begin{tabular}{|c|c|c|c|c|c|c|c|c|c|c|c|c|c|c|c|}
\hline \multirow[t]{2}{*}{ Iras name } & \multirow[t]{2}{*}{$\mathrm{ps}^{2}$} & \multicolumn{2}{|c|}{$\begin{array}{c}V_{b}^{1} \\
\mathrm{~km} \mathrm{~s}^{-1}\end{array}$} & \multicolumn{2}{|c|}{$\begin{array}{c}V_{r}^{1} \\
\mathrm{~km} \mathrm{~s}^{-1}\end{array}$} & \multicolumn{2}{|c|}{$\begin{array}{c}V_{\text {int }}^{1,3} \\
\mathrm{~km} \mathrm{~s}^{-1}\end{array}$} & \multirow[t]{2}{*}{$\begin{array}{r}V_{*}^{1} \\
\mathrm{~km} \mathrm{~s}^{-1}\end{array}$} & \multirow[t]{2}{*}{$\begin{array}{r}V_{\text {exp }}^{1} \\
\mathrm{~km} \mathrm{~s}^{-1}\end{array}$} & \multicolumn{2}{|c|}{$\begin{array}{c}S_{b}^{1} \\
\mathrm{Jy}\end{array}$} & \multicolumn{2}{|c|}{$\begin{array}{l}S_{r}^{1} \\
\mathrm{Jy}\end{array}$} & \multicolumn{2}{|c|}{$\begin{array}{c}S_{\text {int }}^{1,3} \\
\text { Jy }\end{array}$} \\
\hline & & $\mathrm{L}^{1}$ & $\mathrm{R}^{1}$ & $\mathrm{~L}$ & $\mathrm{R}$ & $\mathrm{L}$ & $\mathrm{R}$ & & & $\mathrm{L}$ & $\mathrm{R}$ & $\mathrm{L}$ & $\mathrm{R}$ & $\mathrm{L}$ & $\mathrm{R}$ \\
\hline $15226-3603$ & & $\ldots$ & $\ldots$ & $\ldots$ & $\ldots$ & & & & & $\ldots$ & $\ldots$ & $\ldots$ & $\ldots$ & & \\
\hline $15262+0400$ & & 37.82 & 37.82 & 50.18 & 50.74 & & & 44.1 & 6.3 & 0.30 & 0.31 & 0.81 & 0.69 & & \\
\hline 20396-0826 & & $\ldots$ & $-53.37 ?$ & -47.19 & -47.19 & & & $50.3 ?$ & $3.1 ?$ & & $0.07 ?$ & 0.12 & 0.11 & & \\
\hline $20547+0247$ & & $\ldots$ & $\ldots$ & $\ldots$ & -75.19 & & & & & $\ldots$ & $\ldots$ & $\ldots$ & 0.11 & & \\
\hline $22525+6033$ & & -62.12 & -57.63 & -43.02 & -46.39 & & & -52.6 & 9.5 & 0.17 & 0.37 & 0.27 & 0.41 & & \\
\hline & & -56.51 & -53.70 & -45.83 & -47.51 & & & & & 0.48 & 0.75 & 0.16 & 0.37 & & \\
\hline & & -53.70 & $\ldots$ & -44.71 & $\ldots$ & & & & & 0.26 & $\ldots$ & 0.17 & $\ldots$ & & \\
\hline & & -51.45 & $\ldots$ & $\ldots$ & $\ldots$ & & & & & 0.24 & $\ldots$ & $\ldots$ & $\ldots$ & & \\
\hline $17482-2824$ & ci & -10.79 & -10.79 & 31.91 & 31.91 & 15.06 & & 10.6 & 21.3 & 0.43 & 0.35 & 0.83 & 0.84 & 0.11 & \\
\hline $17505-3143$ & & -12.86 & -12.86 & 20.29 & 20.29 & & & 3.7 & 16.6 & 0.38 & 0.33 & 0.48 & 0.46 & & \\
\hline $19075+0921$ & & 5.26 & 4.13 & 19.30 & 20.99 & & & 12.4 & 7.7 & 0.70 & 0.47 & 0.45 & 0.11 & & \\
\hline $19190+1128$ & & 41.14 & 41.14 & 74.29 & 74.29 & & & 57.7 & 16.6 & 0.18 & 0.17 & 0.51 & 0.52 & & \\
\hline $19244+1115$ & $\mathrm{ci}$ & 63.89 & 63.32 & 99.29 & 99.29 & & & 81.4 & 17.8 & 0.58 & 0.47 & 0.33 & 0.32 & & \\
\hline & & 70.63 & 71.19 & $\ldots$ & $\ldots$ & & 79.62 & & & 0.84 & 0.63 & $\ldots$ & $\ldots$ & & 0.23 \\
\hline $17220-2448$ & & $\ldots$ & $\ldots$ & $\ldots$ & $\ldots$ & & & & & $\ldots$ & $\ldots$ & $\ldots$ & $\ldots$ & & \\
\hline $17317-3331$ & $\mathrm{~s}$ & -4.05 & -4.05 & 25.17 & 25.17 & 10.00 & 10.00 & -10.6 & 14.6 & 0.81 & 0.71 & 2.99 & 2.71 & 0.26 & 0.23 \\
\hline $17385-3332$ & ci? & $\ldots$ & $\ldots$ & -223.71 & -223.71 & $-246.18 ?$ & $-246.74 ?$ & & & $\ldots$ & $\ldots$ & 0.38 & 0.31 & $0.09 ?$ & 0.11 ? \\
\hline & & $\ldots$ & $\ldots$ & $\ldots$ & $\ldots$ & & $-236.07 ?$ & & & $\ldots$ & $\ldots$ & $\ldots$ & $\ldots$ & & 0.10 ? \\
\hline $17392-3319$ & & -59.05 & -59.05 & -30.39 & -30.39 & & & -44.7 & 14.3 & 0.27 & 0.29 & 0.44 & 0.45 & & \\
\hline $17411-3154$ & $\mathrm{~s}$ & -41.10 & -41.10 & -4.02 & -4.02 & -22.66 & -22.56 & -22.6 & 18.5 & 18.87 & 16.67 & 13.55 & 12.67 & 1.93 & 1.69 \\
\hline $17418-2713$ & & -29.29 & -29.29 & 3.86 & 3.86 & & & -12.7 & 16.6 & 3.72 & 3.28 & 3.43 & 2.99 & & \\
\hline $17550-2120$ & & -34.92 & -35.49 & -7.95 & -7.95 & & & -21.6 & 13.6 & 0.94 & 0.81 & 1.98 & 1.71 & & \\
\hline $18033-2111$ & & $\ldots$ & $\ldots$ & $\ldots$ & $\ldots$ & & & & & $\ldots$ & $\ldots$ & $\ldots$ & $\ldots$ & & \\
\hline $18135-1456$ & $\mathrm{~s}$ & -13.36 & -13.36 & 15.29 & 15.29 & 1.24 & 1.24 & 1.0 & 14.3 & 0.91 & 0.79 & 7.67 & 6.82 & 0.30 & 0.15 \\
\hline $18198-1249$ & $\mathrm{~s}$ & 32.83 & 32.83 & 63.73 & 63.73 & 48.56 & 48.56 & 48.3 & 15.4 & 0.55 & 0.46 & 0.94 & 0.85 & 0.12 & 0.07 \\
\hline $18257-1000$ & $\mathrm{~s}$ & 95.90 & 95.90 & 132.42 & 132.42 & 114.44 & 114.44 & 114.2 & 18.3 & 2.52 & 2.31 & 0.88 & 0.86 & 0.11 & 0.13 \\
\hline $18460-0254$ & $\mathrm{~s}$ & 77.65 & 77.65 & 120.35 & 120.35 & 99.00 & 99.00 & 99.0 & 21.3 & 1.94 & 1.78 & 6.76 & 6.22 & 0.25 & 0.30 \\
\hline $18488-0107$ & & 55.21 & 55.21 & 96.79 & 96.79 & & & 76.0 & 20.8 & 0.16 & 0.16 & 0.98 & 0.98 & & \\
\hline $19065+0832$ & s? & 36.02 & 36.02 & 70.29 & 70.29 & $53.44 ?$ & $54.07 ?$ & 53.2 & 17.1 & 0.47 & 0.46 & 1.63 & 1.46 & $0.09 ?$ & $0.07 ?$ \\
\hline $19254+1631$ & & -17.10 & -18.23 & 19.98 & 19.98 & & & 1.2 & 18.8 & 0.14 & 0.13 & 0.85 & 0.80 & & \\
\hline $19352+2030$ & ci & -8.70 & -8.70 & 7.04 & 7.04 & -1.39 & -1.39 & -0.8 & 7.9 & 0.13 & 0.12 & 0.17 & 0.19 & 0.75 & 0.52 \\
\hline $22177+5936$ & $\mathrm{~s}$ & -40.05 & -40.05 & -10.83 & -10.83 & -26.00 & 26.56 & -25.4 & 14.6 & 0.37 & 0.33 & 0.42 & 0.34 & 0.06 & 0.06 \\
\hline
\end{tabular}

1, 2 and 3: same as for the previous table. 